Generalized Rayleigh-quotient formulas for the real parts, imaginary parts, and moduli of the eigenvalues of diagonalizable matrices

\title{
L. KoHaupt
}

Beuth University of Technology Berlin, Department of Mathematics, Luxemburger Str. 10, D-13353 Berlin, E-mail: kohaupt@beuth-hochschule.de received 23 April 2018; accepted 21 May 2018 DOI 10.24297/jam.v14i2.7373

\begin{abstract}
In the present paper, generalized Rayleigh-quotient formulas for the real parts, imaginary parts, and moduli of the eigenvalues of diagonalizable matrices are derived. These formulas are new and correspond to similar formulas for the eigenvalues of self-adjoint matrices obtained recently. Numerical examples underpin the theoretical findings.
\end{abstract}

Key words: Diagonalizable matrix; Real and imaginary parts of eigenvalues; Generalized Rayleighquotient formulas; Generalized numerical range; System matrix of a linear dynamical problem

\section{Introduction}

For self-adjoint matrices, there are formulas for the eigenvalues in the form of generalized Rayleigh quotients; more precisely, max-, min-, minmax-, and maxmin-formulas are known; for this, see [7].

The aim of the present paper is to derive corresponding formulas for the real parts, imaginary parts, and moduli of the eigenvalues of diagonalizable matrices. It will turn out that this is indeed possible for the real and imaginary parts. For the moduli, only a max-formula could be obtained, however. We mention that the presentation follows closely that of [7] for the self-adjoint case.

The paper is structured as follows.

In Sections 2 - 4, the new generalized Rayleigh-quotient formulas for the real parts, imaginary parts, and moduli are deduced, as the case may be. In Section 5, the special case of diagonalizable matrices with real eigenvalues is treated. Section 6 contains an application and Section 7 the definitions of new generalized numerical ranges. In Sections 8 and 9, numerical examples are presented that underpin the obtained findings. In the first example, matrix $A$ is taken as the system matrix of a linear dynamical problem. In the second example, we choose a matrix with real eigenvalues. Finally, Section 10 contains the conclusion and an outlook on to future work. The References are restricted to those that are cited in this paper augmented by those used in [7], the latter being [2], [3], [10], and [11].

\section{Generalized Rayleigh-quotient formulas for the real parts of the eigen- values of a diagonalizable matrix}

In this section, we want to derive formulas for the representation of the real parts of the eigenvalues of a diagonalizable matrix $A \in \mathbb{C}^{n \times n}$ by Rayleigh quotients that generalize existing ones. More precisely, max-, min-, minmax-, and maxmin-representations are 
obtained in the form of more general Rayleigh quotients corresponding to associated formulas for the eigenvalues of self-adjoint matrices assembled in [7]. The difference to the results obtained in $[8]$ is that here we use the scalar product $(u, v)$ in $\mathbb{C}^{n}$ instead of a weighted scalar product $(u, v)_{R}$.

First, we formulate the following conditions $(C 1)-(C 4)$ :

(C1) $A \in \mathbb{C}^{n \times n}$

(C2) $\mathrm{A}$ is diagonalizable, and $\lambda_{i}, i=1, \cdots, n$ are the eigenvalues of $A$ as well as $p_{i}, i=1, \cdots, n$ the associated eigenvectors

(C3) $u_{i}^{*}, i=1, \cdots, n$ are the eigenvectors of $A^{*}$ corresponding to the eigenvalues $\lambda_{i}, i=1, \cdots, n$ of $A^{*}$

(C4) $\lambda_{i} \neq \lambda_{j}, i \neq j, i, j=1, \cdots, n$

We mention that, even though condition $(C 4)$ may be omitted (see [6, Theorem 3]), it is nevertheless useful here since it will turn out to be fulfilled in the numerical examples in Sections 8 and 9 and since the biorthogonal system in Theorem 1 can be constructed more easily than without this condition.

Theorem 1: (Biorthogonality relations with $\lambda_{i} \neq \lambda_{j}, i \neq j, i, j=1, \cdots, n$ )

Let the conditions $(C 1)-(C 4)$ be fulfilled. Then, after appropriate normalization of the eigenvectors $p_{i}, i=1, \cdots, n$ and $u_{i}^{*}, i=1, \cdots, n$, one has the biorthogonality relations

$$
\left(p_{i}, u_{j}^{*}\right)=\delta_{i j}, i, j=1, \cdots, n,
$$

where $(\cdot, \cdot)$ is the usual scalar product on $\mathbb{C}^{n}$.

Proof: See [5, Theorem 1] or [6, Theorem 3].

Next, we want to derive a relation corresponding to that of [7, Formula (6)]. This is done in the following Formula (12).

For this, first, we define the following subspaces of $\mathbb{C}^{n}$. For every $k=1, \cdots, n$, let

$$
N_{p, k}:=\left\{u \in \mathbb{C}^{n} \mid u=\sum_{j=1}^{k} \alpha_{j} p_{j} \text { with } \alpha_{j} \in \mathbb{C}, j=1, \cdots, k\right\}=:\left[p_{1}, \cdots, p_{k}\right]
$$

and

$$
N_{p, k, \mathbb{R}}:=\left\{u \in \mathbb{C}^{n} \mid u=\sum_{j=1}^{k} \beta_{j} p_{j} \text { with } \beta_{j} \in \mathbb{R}, j=1, \cdots, k\right\}=:\left[p_{1}, \cdots, p_{k}\right]_{\mathbb{R}}
$$

as well as

$$
N_{p}:=N_{p, n}:=\left\{u \in \mathbb{C}^{n} \mid u=\sum_{j=1}^{n} \alpha_{j} p_{j} \text { with } \alpha_{j} \in \mathbb{C}, j=1, \cdots, n\right\}=:\left[p_{1}, \cdots, p_{n}\right]
$$

and

$N_{p, \mathbb{R}}:=N_{p, n, \mathbb{R}}:=\left\{u \in \mathbb{C}^{n} \mid u=\sum_{j=1}^{n} \beta_{j} p_{j}\right.$ with $\left.\beta_{j} \in \mathbb{R}, j=1, \cdots, n\right\}=:\left[p_{1}, \cdots, p_{n}\right]_{\mathbb{R}}$

where $N_{p, \mathbb{R}}$ is apparently isomorphic to $\mathbb{R}^{n}$ and $N_{p}=\mathbb{C}^{n}$.

Likewise, we define

$$
N_{u^{*}, k}:=\left\{u \in \mathbb{C}^{n} \mid u=\sum_{j=1}^{k} \alpha_{j} u_{j}^{*} \text { with } \alpha_{j} \in \mathbb{C}, j=1, \cdots, k\right\}=:\left[u_{1}^{*}, \cdots, u_{k}^{*}\right]
$$


and

$$
N_{u^{*}, k, \mathbb{R}}:=\left\{u \in \mathbb{C}^{n} \mid u=\sum_{j=1}^{k} \beta_{j} u_{j}^{*} \text { with } \beta_{j} \in \mathbb{R}, j=1, \cdots, k\right\}=:\left[u_{1}^{*}, \cdots, u_{k}^{*}\right]_{\mathbb{R}}
$$

as well as

$$
N_{u^{*}}:=N_{u^{*}, n}:=\left\{u \in \mathbb{C}^{n} \mid u=\sum_{j=1}^{n} \alpha_{j} u_{j}^{*} \text { with } \alpha_{j} \in \mathbb{C}, j=1, \cdots, n\right\}=:\left[u_{1}^{*}, \cdots, u_{n}^{*}\right]
$$

and

$N_{u^{*}, \mathbb{R}}:=N_{u^{*}, n, \mathbb{R}}:=\left\{u \in \mathbb{C}^{n} \mid u=\sum_{j=1}^{n} \beta_{j} u_{j}^{*}\right.$ with $\left.\beta_{j} \in \mathbb{R}, j=1, \cdots, n\right\}=:\left[u_{1}^{*}, \cdots, u_{n}^{*}\right]_{\mathbb{R}}$

where $N_{u^{*}, \mathbb{R}}$ is apparently isomorphic to $\mathbb{R}^{n}$ and $N_{u^{*}}=\mathbb{C}^{n}$.

After these preparations, we are able to prove the following lemma.

\section{Lemma 2:}

Let the conditions (C1) - (C4) be fulfilled. Then, with the denotations of Theorem 1,

$$
(A u, v)=\sum_{j=1}^{n} \lambda_{j}(A)\left(u, u_{j}^{*}\right)\left(p_{j}, v\right), u, v \in \mathbb{C}^{n},
$$

where

$$
\left(u, u_{j}^{*}\right),\left(p_{j}, v\right) \in \mathbb{R}, u \in N_{p, \mathbb{R}}, v \in N_{u^{*}, \mathbb{R}} j=1, \cdots, n
$$

leading to

$$
\operatorname{Re}(A u, v)=\sum_{j=1}^{n} \operatorname{Re} \lambda_{j}(A)\left(u, u_{j}^{*}\right)\left(p_{j}, v\right), u \in N_{p, \mathbb{R}}, v \in N_{u^{*}, \mathbb{R}} .
$$

Proof: Let $u, v \in \mathbb{C}^{n}$. Then,

$$
u=\sum_{j=1}^{n}\left(u, u_{j}^{*}\right) p_{j}
$$

and

$$
v=\sum_{k=1}^{n}\left(v, p_{k}\right) u_{k}^{*}
$$

implying

$$
(A u, v)=\sum_{j, k=1}^{n} \lambda_{j}(A)\left(u, u_{j}^{*}\right) \overline{\left(v, p_{k}\right)}\left(p_{j}, u_{k}^{*}\right)
$$

so that (10) follows.

Further, let $j \in\{1, \cdots, n\}$ and $u \in N_{p, \mathbb{R}}$. Then, $u=\sum_{k=1}^{n} \alpha_{k} p_{k}$ with elements $\alpha_{k} \in \mathbb{R}, k=1, \cdots, n$. Therefore,

$$
\left(u, u_{j}^{*}\right)=\sum_{k=1}^{n} \alpha_{k}\left(p_{k}, u_{j}^{*}\right)=\alpha_{j} \in \mathbb{R} .
$$

Correspondingly, for $v \in N_{u^{*}, \mathbb{R}}$, one has $\left(p_{j}, v\right) \in \mathbb{R}$ so that (11) is proven. Relation (12) is a direct consequence of (10) and (11). 
Next, we want to define vector spaces similar to the spaces $M_{k, \mathbb{R}}, k=1, \cdots, n$ in [7, Section 2)], namely:

$$
\begin{aligned}
& M_{p, 1, \mathbb{R}}:=N_{p, \mathbb{R}}=\left[p_{1}, \cdots, p_{n}\right]_{\mathbb{R}}, \\
& M_{p, k, \mathbb{R}}:=\left\{u \in N_{p, \mathbb{R}} \mid\left(u, u_{j}^{*}\right)=0, j=1,2, \cdots, k-1\right\}, k=2, \cdots, n
\end{aligned}
$$

and

$$
\begin{aligned}
& M_{u^{*}, 1, \mathbb{R}}:=N_{u^{*}, \mathbb{R}}=\left[u_{1}^{*}, \cdots, u_{n}^{*}\right]_{\mathbb{R}}, \\
& M_{u^{*}, k, \mathbb{R}}:=\left\{u \in N_{u^{*}, \mathbb{R}} \mid\left(u, p_{j}\right)=0, j=1,2, \cdots, k-1\right\}, k=2, \cdots, n .
\end{aligned}
$$

The next lemma characterizes these spaces.

\section{Lemma 3:}

Let the conditions $(C 1)-(C 4)$ be fulfilled as well as $\left\{p_{1}, \cdots, p_{n}\right\}$ and $\left\{u_{1}^{*}, \cdots, u_{n}^{*}\right\}$ be a biorthogonal set of eigenvectors of $A$ and $A^{*}$, i.e. such that

$$
\left(p_{i}, u_{j}^{*}\right)=\delta_{i j}, i, j=1, \cdots, n .
$$

Then,

and

$$
M_{p, k, \mathbb{R}}=\left[p_{k}, p_{k+1}, \cdots, p_{n}\right]_{\mathbb{R}}, k=1, \cdots, n
$$

$$
M_{u^{*}, k, \mathbb{R}}=\left[u_{k}^{*}, u_{k+1}^{*}, \cdots, u_{n}^{*}\right]_{\mathbb{R}}, k=1, \cdots, n .
$$

Proof: The proof is done for (18) and $k=3$. The general case can be made by induction. The proof for (19) is similar. So, we have to prove

$$
M_{p, 3, \mathbb{R}}=\left\{u \in N_{p, \mathbb{R}} \mid\left(u, u_{1}^{*}\right)=0,\left(u, u_{2}^{*}\right)=0\right\}=\left[p_{3}, p_{4}, \cdots, p_{n}\right]_{\mathbb{R}} .
$$

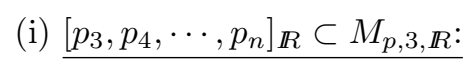

Let $u \in\left[p_{3}, p_{4}, \cdots, p_{n}\right]_{\mathbb{R}}$. Then, $u=\sum_{j=3}^{n} \beta_{j} p_{j}$ with elements $\beta_{j} \in \mathbb{R}, j=3, \cdots, n$. Let $s \in\{1,2\}$. This entails, due to Theorem $1,\left(u, u_{j}^{*}\right)=\sum_{k=3}^{n} \beta_{k}\left(p_{k}, u_{j}^{*}\right)=0$ so that (i) is proven.

(ii) $\underline{M_{p, 3, \mathbb{R}} \subset\left[p_{3}, p_{4}, \cdots, p_{n}\right]_{\mathbb{R}}}$ :

Let $u \in M_{p, 3, \mathbb{R}}$. This implies $u \in N_{p, \mathbb{R}}$ and $\left(u, u_{j}^{*}\right)=0, j=1,2$. Now, $u=\sum_{k=1}^{n} \beta_{k} p_{k}$ with $\beta_{k}=\left(u, u_{k}^{*}\right) \in \mathbb{R}, k=1, \cdots, n$ leading to $u=\sum_{k=3}^{n} \beta_{k} p_{k}$ since $\left(u, u_{k}^{*}\right)=0, k=$ 1,2 so that (ii) follows.

Similarly to $[7,(11)]$, we suppose that the eigenvalues $\lambda_{1}(A), \cdots, \lambda_{n}(A)$ of matrix $A$ are arranged such that

$$
\operatorname{Re} \lambda_{1}(A) \geq \operatorname{Re}_{2}(A) \geq \cdots \geq \operatorname{Re} \lambda_{n}(A) .
$$

Further, let $u \in N_{p, \mathbb{R}}$ with $u=\sum_{k=1}^{n} \alpha_{k} p_{k}$ and $v \in N_{u^{*}, \mathbb{R}}$ with $v=\sum_{k=1}^{n} \beta_{k} u_{k}^{*}$. Then due to Theorem 1,

$$
(u, v)=\sum_{k=1}^{n} \alpha_{k} \beta_{k} .
$$

In order to facilitate the manner of speaking, we say that the scalar product $(u, v)$ of $u$ and $v$ is strongly positive if $\alpha_{k} \beta_{k} \geq 0, k=1, \cdots, n$ and $\sum_{k=1}^{n} \alpha_{k} \beta_{k}>0$. For short, we write $(u, v) \gg 0$.

Remark: One has $\alpha_{k}=\left(u, u_{k}^{*}\right), u \in N_{p, \mathbb{R}}$ and $\beta_{k}=\left(p_{k}, v\right), v \in N_{u^{*}, \mathbb{R}}$ for $k=$ $1, \cdots, n$. Therefore, $(u, v) \gg 0$ means $\left(u, u_{k}^{*}\right)\left(p_{k}, v\right) \geq 0, k=1, \cdots, n$ and $(u, v)=$ $\sum_{k=1}^{n}\left(u, u_{k}^{*}\right)\left(p_{k}, v\right)>0$. 
Remark: More generally, in the sequel, one could admit linear combinations $u=$ $\sum_{k=1}^{n} \alpha_{k} p_{k}$ and $v=\sum_{k=1}^{n} \beta_{k} u_{k}^{*}$ with $\alpha_{k}, \beta_{k} \in \mathbb{C}$ such that $\alpha_{k} \overline{\beta_{k}}=\left|\alpha_{k} \beta_{k}\right|$ and $\sum_{k=1}^{n}\left|\alpha_{k} \beta_{k}\right|>0$. For example, all elements $\alpha_{k}, \beta_{k} \in \mathbb{C}$ with $\alpha_{k}=\left|\alpha_{k}\right| e^{i \varphi_{k}}$ and $\beta_{k}=\left|\beta_{k}\right| e^{i \varphi_{k}}$ where $\varphi_{k}$ is in $0 \leq \varphi_{k}<2 \pi$ for $k=1, \cdots, n$ would be acceptable. But, we do not want to pursue this aspect in more detail.

One has the following generalized max-representation.

\section{Theorem 4:}

Let the conditions $(C 1)-(C 4)$ be fulfilled. Further, let the eigenvalues of $A$ be arranged according to (21). Moreover, let the vector spaces $M_{p, k, \mathbb{R}}$ and $M_{u^{*}, k, \mathbb{R}}$ for $k=1, \cdots, n$ be defined by (16), (17) or (18),(19).

Then,

$$
\operatorname{Re}_{k}(A)=\max _{\substack{(u, v) \gg 0 \\ u \in M_{p, k}, \mathbb{R}, v \in M_{u^{*}, k, \mathbb{R}}}} \frac{\operatorname{Re}(A u, v)}{(u, v)}, k=1,2, \cdots, n .
$$

The maximum is attained for $u=p_{k}, v=u_{k}^{*}$.

Proof: One uses equation (12) as starting point, i.e.,

$$
\operatorname{Re}(A u, v)=\sum_{j=1}^{n} \operatorname{Re} \lambda_{j}\left(u, u_{j}^{*}\right)\left(p_{j}, v\right), u \in N_{p, \mathbb{R}}, v \in N_{u^{*}, \mathbb{R}}
$$

with

$$
R e \lambda_{j},\left(u, u_{j}^{*}\right),\left(p_{j}, v\right) \in \mathbb{R}, u \in N_{p, \mathbb{R}}, v \in N_{u^{*}, \mathbb{R}}, j=1, \cdots, n .
$$

Let $k \in\{1, \cdots, n\}$ be arbitrarily chosen, but fixed and $u \in M_{p, k, \mathbb{R}} \subset N_{p, \mathbb{R}}$ and $v \in M_{u^{*}, k, \mathbb{R}} \subset N_{u^{*}, \mathbb{R}}$ also arbitrarily chosen, but fixed with $(u, v) \gg 0$. Then,

$$
\begin{aligned}
\operatorname{Re}(A u, v) & =\sum_{j=k}^{n} \operatorname{Re} \lambda_{j}\left(u, u_{j}^{*}\right)\left(p_{j}, v\right) \leq \max _{j=k, \cdots, n} \operatorname{Re} \lambda_{j} \sum_{j=k}^{n}\left(u, u_{j}^{*}\right)\left(p_{j}, v\right) \\
& =\operatorname{Re} \lambda_{k} \sum_{j=1}^{n}\left(u, u_{j}^{*}\right)\left(p_{j}, v\right),
\end{aligned}
$$

that is,

$$
\frac{\operatorname{Re}(A u, v)}{(u, v)} \leq \operatorname{Re} \lambda_{k}(A)
$$

and thus

$$
\max _{\substack{(u, v) \gg 0 \\ u \in M_{p, k}, \mathbb{R}, v \in M_{u^{*}, k, \mathbb{R}}}} \frac{\operatorname{Re}(A u, v)}{(u, v)} \leq \operatorname{Re} \lambda_{k}(A) .
$$

Now, $\operatorname{Re} \lambda_{k}(A)$ is attained for $u=p_{k} \in M_{p, k, \mathbb{R}}$ and $v \in M_{u^{*}, k, \mathbb{R}}$, that is,

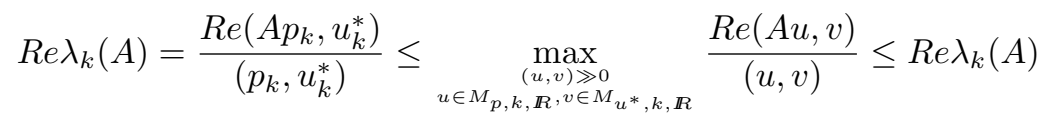

so that (22) is proven.

$\diamond$

From the proof of Theorem 4, it seems that one can carry over many results from the self-adjoint case handled in [7] to the case of diagonalizable matrices. So, we have the following further generalized Rayleigh-quotient representations for $\operatorname{Re} \lambda_{j}(A)$ instead of $\lambda_{j}(A)$ corresponding to [7, Theorem5].

\section{Theorem 5:}

Let the conditions (C1) - (C4) be fulfilled. Further, let the eigenvalues of $A$ be arranged according to (21). 
Then, for every $j=1, \cdots, n$ and every subspace $M_{p} \subset N_{p, \mathbb{R}}$ and $M_{u^{*}} \subset N_{u^{*}, \mathbb{R}}$ with $\operatorname{dim} M_{p}=\operatorname{dim} M_{u^{*}}=m=n+1-j$, the following inequalities are valid:

$$
\operatorname{Re}_{j}(A) \leq \max _{\substack{(u, v)>0 \\ u \in M_{p}, v \in M_{u^{*}}}} \frac{\operatorname{Re}(A u, v)}{(u, v)} \leq \operatorname{Re} \lambda_{1}(A),
$$

and the following representation formulas hold:

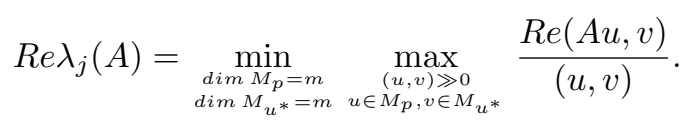

Proof: One has

$$
\operatorname{Re}_{1}(A)=\max _{\substack{(u, v) \gg 0 \\ u \in N_{p}, \mathbb{R}, v \in N_{u^{*}, \mathbb{R}}}} \frac{(A u, v)}{(u, v)} \geq \max _{\substack{(u, v)>0 \\ u \in M_{p}, v \in M_{u^{*}}}} \frac{\operatorname{Re}(A u, v)}{(u, v)},
$$

for all subspaces $M_{p} \subset N_{p, \mathbb{R}}$ and $M_{u^{*}} \subset N_{u^{*}, \mathbb{R}}$ with $\operatorname{dim} M_{p}=\operatorname{dim} M_{u^{*}}=m=$ $n+1-j$.

Determination of subspaces $M_{p} \subset N_{p, \mathbb{R}}$ and $M_{u^{*}} \subset N_{u^{*}, \mathbb{R}}$ with $\operatorname{dim} M_{p}=\operatorname{dim} M_{u^{*}}$ $=m=n+1-j=n-(j-1)$ and of elements $0 \neq z_{p} \in M_{p}$ and $0 \neq z_{u^{*}} \in M_{u^{*}}$ with $\underline{\left(z_{p}, z_{u^{*}}\right) \gg 0 \text { and } \operatorname{Re}\left(A z_{p}, z_{u^{*}}\right) \geq \operatorname{Re} \lambda_{j}\left(z_{p}, z_{u^{*}}\right)}$

In $N_{p, \mathbb{R}}$, there is a complete orthonormal system $v_{1}, v_{2}, \cdots, v_{n}$; herewith, let

$$
M_{p}:=\left[v_{1}, \cdots, v_{m}\right]_{\mathbb{R}} \text { and } M_{p}^{\perp}=\left[v_{m+1}, \cdots, v_{n}\right]_{\mathbb{R}} .
$$

Let

$$
z_{p}=\sum_{i=1}^{j} \alpha_{i} p_{i}
$$

where the elements $\alpha_{1}, \cdots, \alpha_{j} \in \mathbb{R}$ are determined such that

$$
\left(z_{p}, v_{k}\right)=0, k=m+1, \cdots, n .
$$

This entails

$$
z_{p} \in\left[v_{m+1}, \cdots, v_{n}\right]_{\mathbb{R}}^{\perp}=M_{p} \text { and } \operatorname{dim} M_{p}=m
$$

From (29),

$$
\sum_{i=1}^{j} \alpha_{i}\left(p_{i}, v_{k}\right)=0, k=m+1, \cdots, n .
$$

This is a linear system with real coefficients $\left(p_{i}, v_{k}\right)$ as well as $j$ unknowns and $n-m=$ $j-1$ equations. Thus, a nontrivial real solution $\alpha_{1}, \cdots, \alpha_{j}$ exists meaning that

$$
z_{p} \neq 0 .
$$

Define

$$
z_{u^{*}}=\sum_{i=1}^{j} \alpha_{i} u_{i}^{*}
$$

with the same components $\alpha_{i}, i=1, \cdots, j$ as in (28). Then also

$$
z_{u_{i}^{*}} \neq 0 \text {. }
$$

Further,

$$
\left(z_{p}, z_{u^{*}}\right)=\sum_{i=1}^{j} \alpha_{i}^{2}>0
$$


so that $\left(z_{p}, z_{u^{*}}\right) \gg 0$. Moreover,

$$
z_{u^{*}} \in\left[z_{u^{*}}\right] \subset M_{u^{*}}
$$

where $M_{u^{*}}$ is any subspace of $N_{u^{*}, \mathbb{R}}$ with dimension $m$ containing the element $z_{u^{*}}$. From the above, it follows

$$
\left(A z_{p}, z_{u^{*}}\right)=\sum_{i, k=1}^{j} \alpha_{i} \lambda_{i} \alpha_{k}\left(p_{i}, u_{k}^{*}\right)=\sum_{i=1}^{j} \lambda_{i} \alpha_{i}^{2}
$$

Now,

$$
\alpha_{i}=\left(z_{p}, u_{i}^{*}\right) \in \mathbb{R}, i=1, \cdots, j
$$

Therefore,

$$
\operatorname{Re}\left(A z_{p}, z_{u^{*}}\right) \geq \min _{i=1, \cdots, j} \operatorname{Re} \lambda_{i} \sum_{i=1}^{j} \alpha_{i}^{2}=\operatorname{Re} \lambda_{j} \sum_{i=1}^{j} \alpha_{i}^{2}=\operatorname{Re} \lambda_{j}\left(z_{p}, z_{u^{*}}\right)
$$

leading to

$$
\frac{\operatorname{Re}\left(A z_{p}, z_{u^{*}}\right)}{\left(z_{p}, z_{u^{*}}\right)} \geq \operatorname{Re} \lambda_{j}
$$

Further, according to (12),

$$
\operatorname{Re}(A u, v) \leq \max _{j=1, \cdots, n} \operatorname{Re} \lambda_{j} \sum_{j=1}^{n}\left(u, u_{j}^{*}\right)\left(p_{j}, v\right)=\operatorname{Re} \lambda_{1}(u, v),
$$

$(u, v) \gg 0, u \in N_{p, \mathbb{R}}, v \in N_{u^{*}, \mathbb{R}}$ so that

$$
R e \lambda_{1} \geq \frac{\operatorname{Re}(A u, v)}{(u, v)},(u, v) \gg 0, u \in N_{p, \mathbb{R}}, v \in N_{u^{*}, \mathbb{R}}
$$

This implies

$$
\operatorname{Re} \lambda_{j} \leq \frac{\operatorname{Re}\left(A z_{p}, z_{u^{*}}\right)}{\left(z_{p}, z_{u^{*}}\right)} \leq \max _{\substack{(u, v) \gg 0 \\ u \in M_{p}, v \in M_{u^{*}}}} \frac{\operatorname{Re}(A u, v)}{(u, v)} \leq \operatorname{Re} \lambda_{1} .
$$

Therefore, (24) is proven.

From (38), we conclude

$$
\min _{\substack{\operatorname{dim} M_{p}=m \\ \operatorname{dim} M_{u^{*}}=m}} \max _{\substack{(u, v) \gg 0 \\ u \in M_{p}, v \in M_{u^{*}}}} \frac{\operatorname{Re}(A u, v)}{(u, v)} \geq \operatorname{Re} \lambda_{j} .
$$

On the other hand, from Theorem 4,

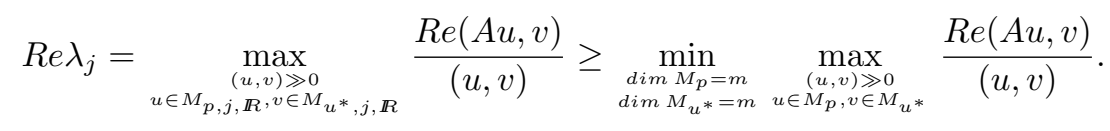

Relations (39) and (40) imply (25).

Remark: From (24), it follows

$$
\frac{\operatorname{Re}(A u, v)}{(u, v)} \leq \nu[A]=\max _{j=1, \cdots, n} \operatorname{Re} \lambda_{j}(A),(u, v) \gg 0, u \in N_{p, \mathbb{R}}, v \in N_{u^{*}, \mathbb{R}} .
$$

The next theorem contains a generalized min-representation of $R e \lambda_{k}$. 


\section{Theorem 6:}

Let the conditions $(C 1)-(C 4)$ be fulfilled. Further, let the eigenvalues of $A$ be arranged according to (21). Moreover, let the vector spaces $N_{p, k, \mathbb{R}}$ and $N_{u^{*}, k, \mathbb{R}}$ for $k=1, \cdots, n$ be defined by (3) and (7).

Then,

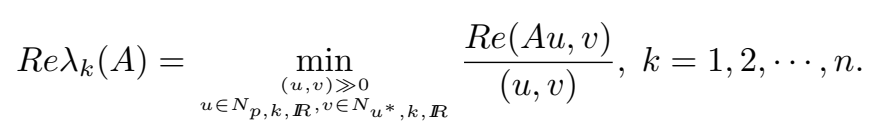

The minimum is attained for $u=p_{k}, v=u_{k}^{*}$.

Proof: According to (12),

$$
\operatorname{Re}(A u, v)=\sum_{j=1}^{n} \operatorname{Re} \lambda_{j}(A)\left(u, u_{j}^{*}\right)\left(p_{j}, v\right), u \in N_{p, \mathbb{R}}, v \in N_{u^{*}, \mathbb{R}}
$$

with

$$
\operatorname{Re} \lambda_{j},\left(u, u_{j}^{*}\right),\left(p_{j}, v\right) \in \mathbb{R}, u \in N_{p, \mathbb{R}}, v \in N_{u^{*}, \mathbb{R}}, j=1, \cdots, n .
$$

Let $k \in\{1, \cdots, n\}$ be arbitrarily chosen, but fixed and $u \in N_{p, k, \mathbb{R}} \subset N_{p, \mathbb{R}}$ and $v \in N_{u^{*}, k, \mathbb{R}} \subset N_{u^{*}, \mathbb{R}}$ also arbitrarily chosen, but fixed with $(u, v) \gg 0$. Then, with $(21)$,

$$
\begin{aligned}
\operatorname{Re}(A u, v) & =\sum_{j=1}^{k} \operatorname{Re} \lambda_{j}\left(u, u_{j}^{*}\right)\left(p_{j}, v\right) \geq \min _{j=1, \cdots, k} \operatorname{Re} \lambda_{j} \sum_{j=1}^{k}\left(u, u_{j}^{*}\right)\left(p_{j}, v\right) \\
& =\operatorname{Re} \lambda_{k}(u, v),
\end{aligned}
$$

that is,

$$
\frac{\operatorname{Re}(A u, v)}{(u, v)} \geq \operatorname{Re} \lambda_{k},(u, v) \gg 0, u \in N_{p, k, \mathbb{R}}, v \in N_{u^{*}, k, \mathbb{R}}
$$

and therefore

$$
\min _{\substack{(u, v) \gg 0 \\ u \in N_{p, k}, \mathbb{R}, v \in N_{u^{*}, k, \mathbb{R}}}} \frac{\operatorname{Re}(A u, v)}{(u, v)} \geq \operatorname{Re} \lambda_{k} .
$$

Now, $\operatorname{Re} \lambda_{k}(A)$ is attained for $u=p_{k} \in N_{p, k, \mathbb{R}}$ and $v=u_{k}^{*} \in N_{u^{*}, k, \mathbb{R}}$; one has $\left(p_{k}, u_{k}^{*}\right) \gg 0$ and

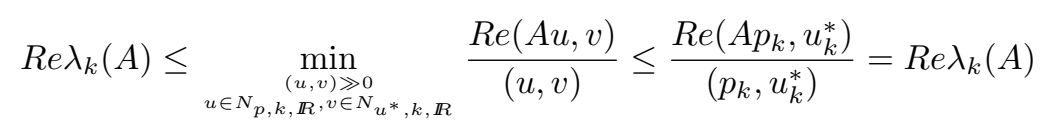

so that the assertion (41) follows.

Correspondingly to $[7$, Theorem 7$]$, we obtain the following generalized maxminrepresentaion of $\operatorname{Re} \lambda_{j}$.

\section{Theorem 7:}

Let the conditions (C1) - (C4) be fulfilled. Further, let the eigenvalues of A be arranged according to (21).

Then, for every $j=1, \cdots, n$ and all subspaces $N_{p} \subset N_{p, \mathbb{R}}$ and $N_{u^{*}} \subset N_{u^{*}, \mathbb{R}}$ with $\operatorname{dim} N_{p}=\operatorname{dim} N_{u^{*}}=j$, the following inequalities are valid:

$$
\operatorname{Re} \lambda_{n}(A) \leq \min _{\substack{(u, v)>0 \\ u \in N_{p}, v \in N_{u^{*}}}} \frac{\operatorname{Re}(A u, v)}{(u, v)} \leq \operatorname{Re} \lambda_{j}(A)
$$


and the following representation formulas hold:

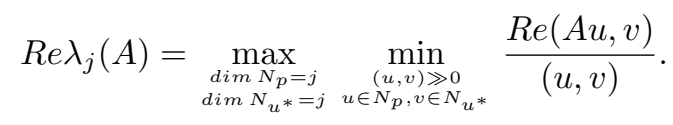

Proof: One has

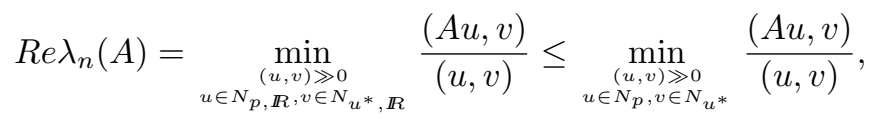

for all subspaces $N_{p} \subset N_{p, \mathbb{R}}$ and $N_{u^{*}} \subset N_{u^{*}, \mathbb{R}}$ with $\operatorname{dim} N_{p}=\operatorname{dim} N_{u^{*}}=j$.

Determination of subspaces $N_{p} \subset N_{p, \mathbb{R}}$ and $N_{u^{*}} \subset N_{u^{*}, \mathbb{R}}$ with $\operatorname{dim} N_{p}=\operatorname{dim} N_{u^{*}}=j$ and of elements $0 \neq z_{p} \in N_{p}$ and $0 \neq z_{u^{*}} \in N_{u^{*}}$ with $\left(z_{p}, z_{u^{*}}\right) \gg 0$ and $\underline{\operatorname{Re}\left(A z_{p}, z_{u^{*}}\right) \leq \operatorname{Re} \lambda_{j}\left(z_{p}, z_{u^{*}}\right)}$

In $N_{p, \mathbb{R}}$, there is a complete orthonormal system $q_{1}, q_{2}, \cdots, q_{n}$; herewith, let

$$
N_{p}:=\left[q_{1}, \cdots, q_{j}\right]_{\mathbb{R}} \text { and } N_{p}^{\perp}=\left[q_{j+1}, \cdots, q_{n}\right]_{\mathbb{R}} .
$$

Let

$$
z_{p}=\sum_{i=j}^{n} \alpha_{i} p_{i}
$$

where the elements $\alpha_{j}, \cdots, \alpha_{n} \in \mathbb{R}$ are determined such that

$$
\left(z_{p}, q_{k}\right)=0, k=j+1, \cdots, n .
$$

This entails

$$
z_{p} \in\left[q_{j+1}, \cdots, q_{n}\right]_{\mathbb{R}}^{\perp}=N_{p} \text { and } \operatorname{dim} N_{p}=j .
$$

From (48),

$$
\sum_{i=j}^{n} \alpha_{i}\left(p_{i}, q_{k}\right)=0, k=j+1, \cdots, n .
$$

This is a linear system with real coefficients $\left(p_{i}, q_{k}\right)$ as well as $n-(j-1)=n-j+1$ unknowns and $n-j$ equations. Thus, a nontrivial real solution $\alpha_{j}, \cdots, \alpha_{n}$ exists meaning that

$$
z_{p} \neq 0 .
$$

Define

$$
z_{u^{*}}=\sum_{i=j}^{n} \alpha_{i} u_{i}^{*}
$$

with the same components $\alpha_{i}, i=j, \cdots, n$ as in (47). Then also

$$
z_{u_{i}^{*}} \neq 0
$$

Further,

$$
\left(z_{p}, z_{u^{*}}\right)=\sum_{i=j}^{n} \alpha_{i}^{2}>0
$$

so that $\left(z_{p}, z_{u^{*}}\right) \gg 0$. Moreover,

$$
z_{u^{*}} \in\left[z_{u^{*}}\right] \subset N_{u^{*}}
$$

where $N_{u^{*}}$ is any subspace of $N_{u^{*}, \mathbb{R}}$ with dimension $j$ containing the element $z_{u^{*}}$. 
From the above, it follows

$$
\left(A z_{p}, z_{u^{*}}\right)=\sum_{i, k=j}^{n} \alpha_{i} \lambda_{i} \alpha_{k}\left(p_{i}, u_{k}^{*}\right)=\sum_{i=j}^{n} \lambda_{i} \alpha_{i}^{2} .
$$

Now,

$$
\alpha_{i}=\left(z_{p}, u_{i}^{*}\right) \in \mathbb{R}, i=j, \cdots, n .
$$

Therefore,

$$
\operatorname{Re}\left(A z_{p}, z_{u^{*}}\right) \leq \max _{i=j, \cdots, n} \operatorname{Re} \lambda_{i} \sum_{i=j}^{n} \alpha_{i}^{2}=\operatorname{Re} \lambda_{j} \sum_{i=j}^{n} \alpha_{i}^{2}=\operatorname{Re} \lambda_{j}\left(z_{p}, z_{u^{*}}\right)
$$

leading to

$$
\frac{\operatorname{Re}\left(A z_{p}, z_{u^{*}}\right)}{\left(z_{p}, z_{u^{*}}\right)} \leq \operatorname{Re} \lambda_{j}
$$

Further, according to (12),

$$
\operatorname{Re}(A u, v) \geq \min _{j=1, \cdots, n} \operatorname{Re} \lambda_{j} \sum_{j=1}^{n}\left(u, u_{j}^{*}\right)\left(p_{j}, v\right)=\operatorname{Re} \lambda_{n}(u, v),
$$

$(u, v) \gg 0, u \in N_{p, \mathbb{R}}, v \in N_{u^{*}, \mathbb{R}}$ so that

$$
\operatorname{Re} \lambda_{n} \leq \frac{\operatorname{Re}(A u, v)}{(u, v)},(u, v) \gg 0, u \in N_{p, \mathbb{R}}, v \in N_{u^{*}, \mathbb{R}} .
$$

This implies

$$
\operatorname{Re} \lambda_{j} \geq \frac{\operatorname{Re}\left(A z_{p}, z_{u^{*}}\right)}{\left(z_{p}, z_{u^{*}}\right)} \geq \min _{\substack{u, v)>0 \\ u \in N_{p}, v \in N_{u^{*}}}} \frac{\operatorname{Re}(A u, v)}{(u, v)} \geq \operatorname{Re} \lambda_{n}
$$

Therefore, (43) is proven.

From (55), we conclude

$$
\max _{\substack{\operatorname{dim} N_{p}=j \\ \operatorname{dim} N_{u^{*}=j}=j}} \min _{\substack{(u, v)>0 \\ u \in N_{p}, v \in N_{u^{*}}}} \frac{\operatorname{Re}(A u, v)}{(u, v)} \leq \operatorname{Re} \lambda_{j} .
$$

On the other hand, from Theorem 6 ,

$$
\operatorname{Re} \lambda_{j}=\min _{\substack{(u, v)>0 \\ u \in N_{p}, j, \mathbb{R}, v \in N_{u^{*}, j, \mathbb{R}}}} \frac{\operatorname{Re}(A u, v)}{(u, v)} \leq \max _{\substack{\operatorname{dim} N_{p}=j \\ \operatorname{dim} N_{u^{*}}=j}} \min _{\substack{(u, v) \gg 0 \\ u \in N_{p}, v \in N_{u^{*}}}} \frac{\operatorname{Re}(A u, v)}{(u, v)} .
$$

Relations (56) and (57) imply (44).

Remark: From (43), it follows

$$
\frac{\operatorname{Re}(A u, v)}{(u, v)} \geq-\nu[-A]=\min _{j=1, \cdots, n} \operatorname{Re} \lambda_{j}(A),(u, v) \gg 0, u \in N_{p, \mathbb{R}}, v \in N_{u^{*}, \mathbb{R}} .
$$

\section{Generalized Rayleigh-quotient formulas for the imaginary parts of the eigenvalues of a diagonalizable matrix}

In this section, we want to state formulas for the representation of the imaginary parts of the eigenvalues of a diagonalizable matrix $A \in \mathbb{C}^{n \times n}$ by Rayleigh quotients that generalize existing ones. More precisely, max-, min-, minmax-, and maxminrepresentations are obtained corresponding to those in Section 2.

First, we want to state a relation corresponding to that of (12). 


\section{Lemma 8:}

Let the conditions $(C 1)-(C 4)$ be fulfilled. Then, with the denotations of Theorem 1,

$$
\operatorname{Im}(A u, v)=\sum_{j=1}^{n} \operatorname{Im} \lambda_{j}(A)\left(u, u_{j}^{*}\right)\left(p_{j}, v\right), u \in N_{p, \mathbb{R}}, v \in N_{u^{*}, \mathbb{R}} .
$$

Proof: Equation (58) follows directly from Lemma 2, Formulas (10) and (11)

Similarly to (21), we suppose that the eigenvalues $\lambda_{1}(A), \cdots, \lambda_{n}(A)$ of matrix $A$ are arranged such that

$$
\operatorname{Im} \lambda_{1}(A) \geq \operatorname{Im} \lambda_{2}(A) \geq \cdots \geq \operatorname{Im} \lambda_{n}(A) .
$$

Then, one has a series of theorems for the imaginary parts of the eigenvalues corresponding to those of Theorems $4-7$ in Section 2. The theorems are stated without proofs since the only difference is that Lemma 8 is used instead of Lemma 2.

\section{Theorem 9:}

Let the conditions (C1) - (C4) be fulfilled. Further, let the eigenvalues of $A$ be arranged according to (59). Moreover, let the vector spaces $M_{p, k, \mathbb{R}}$ and $M_{u^{*}, k, \mathbb{R}}$ for $k=1, \cdots, n$ be defined by (16), (17) or (18),(19).

Then,

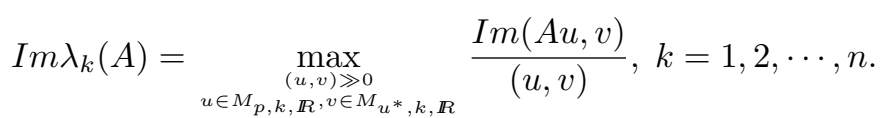

The maximum is attained for $u=p_{k}, v=u_{k}^{*}$.

\section{Theorem 10:}

Let the conditions (C1) - (C4) be fulfilled. Further, let the eigenvalues of A be arranged according to (59).

Then, for every $j=1, \cdots, n$ and every subspace $M_{p} \subset N_{p, \mathbb{R}}$ and $M_{u^{*}} \subset N_{u^{*}, \mathbb{R}}$ with $\operatorname{dim} M_{p}=\operatorname{dim} M_{u^{*}}=m=n+1-j$, the following inequalities are valid:

$$
\operatorname{Im} \lambda_{j}(A) \leq \max _{\substack{(u, v)>0 \\ u \in M_{p}, v \in M_{u^{*}}}} \frac{\operatorname{Im}(A u, v)}{(u, v)} \leq \operatorname{Im} \lambda_{1}(A),
$$

and the following representation formulas hold:

$$
\operatorname{Im} \lambda_{j}(A)=\min _{\substack{\operatorname{dim} M_{p}=m \\
\operatorname{dim} M_{u^{*}=m}}} \max _{\begin{array}{c}
(u, v) \gg 0 \\
u \in M_{p}, v \in M_{u^{*}}
\end{array}} \frac{\operatorname{Im}(A u, v)}{(u, v)} .
$$

Remark: From (61), it follows

$$
\frac{\operatorname{Im}(A u, v)}{(u, v)} \leq \max _{j=1, \cdots, n} \operatorname{Im} \lambda_{j}(A),(u, v) \gg 0, u \in N_{p, \mathbb{R}}, v \in N_{u^{*}, \mathbb{R}} .
$$

\section{Theorem 11:}

Let the conditions $(C 1)-(C 4)$ be fulfilled. Further, let the eigenvalues of $A$ be arranged according to (59). Moreover, let the vector spaces $N_{p, k, \mathbb{R}}$ and $N_{u^{*}, k, \mathbb{R}}$ for $k=1, \cdots, n$ be defined by (3) and (7). 
Then,

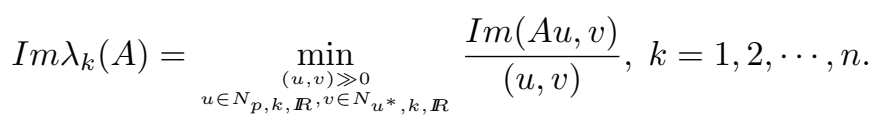

The minimum is attained for $u=p_{k}, v=u_{k}^{*}$.

\section{Theorem 12:}

Let the conditions (C1) - (C4) be fulfilled. Further, let the eigenvalues of $A$ be arranged according to (59).

Then, for every $j=1, \cdots, n$ and all subspaces $N_{p} \subset N_{p, \mathbb{R}}$ and $N_{u^{*}} \subset N_{u^{*}, \mathbb{R}}$ with $\operatorname{dim} N_{p}=\operatorname{dim} N_{u^{*}}=j$, the following inequalities are valid:

$$
\operatorname{Im} \lambda_{n}(A) \leq \min _{\substack{(u, v) \gg 0 \\ u \in N_{p}, v \in N_{u^{*}}}} \frac{\operatorname{Im}(A u, v)}{(u, v)} \leq \operatorname{Im} \lambda_{j}(A),
$$

and the following representation formulas hold:

$$
\operatorname{Im} \lambda_{j}(A)=\max _{\substack{\operatorname{dim} N_{p}=j \\ \operatorname{dim} N_{u^{*}}=j}} \min _{\substack{(u, v) \gg 0 \\ u \in N_{p}, v \in N_{u^{*}}}} \frac{\operatorname{Im}(A u, v)}{(u, v)} .
$$

Remark: From (64), it follows

$$
\frac{\operatorname{Im}(A u, v)}{(u, v)} \geq \min _{j=1, \cdots, n} \operatorname{Im} \lambda_{j}(A),(u, v) \gg 0, u \in N_{p, \mathbb{R}}, v \in N_{u^{*}, \mathbb{R}} .
$$

\section{Generalized Rayleigh-quotient formula for the moduli of the eigenvalues of a diagonalizable matrix}

Whereas in Sections 2 and 3 max-, min-, minmax-, and maxmin-representations with generalized Rayleigh quotients for diagonalizable matrices could be obtained, it seems that, for the moduli of eigenvalues, only a max-representation is possible.

We first deduce this max-representation. For this, we suppose that the eigenvalues $\lambda_{1}(A), \cdots, \lambda_{n}(A)$ of $A \in \mathbb{C}^{n \times n}$ are arranged such that

$$
\left|\lambda_{1}(A)\right| \geq\left|\lambda_{2}(A)\right| \geq \cdots \geq\left|\lambda_{n}(A)\right| \text {. }
$$

Herewith, one has

\section{Theorem 13:}

Let the conditions $(C 1)-(C 4)$ be fulfilled. Further, let the eigenvalues of $A$ be arranged according to (66). Moreover, let the vector spaces $M_{p, k, \mathbb{R}}$ and $M_{u^{*}, k, \mathbb{R}}$ for $k=1, \cdots, n$ be defined by (16), (17) or (18),(19).

Then,

$$
\left|\lambda_{k}(A)\right|=\max _{\substack{(u, v) \gg 0 \\ u \in M_{p, k, \mathbb{R}, v \in M_{u}, k, \mathbb{R}}}} \frac{|(A u, v)|}{(u, v)}, k=1,2, \cdots, n .
$$

The maximum is attained for $u=p_{k}, v=u_{k}^{*}$.

Proof: One uses (10) and (11) as starting point. This leads to

$$
(A u, v)=\sum_{j=1}^{n} \lambda_{j}\left(u, u_{j}^{*}\right)\left(p_{j}, v\right), u, v \in \mathbb{C}^{n} .
$$


Let $k \in\{1, \cdots, n\}$ be arbitrarily chosen, but fixed and $u \in M_{p, k, \mathbb{R}} \subset N_{p, \mathbb{R}}$ and $v \in M_{u^{*}, k, \mathbb{R}} \subset N_{u^{*}, \mathbb{R}}$ also arbitrarily chosen, but fixed with $(u, v) \gg 0$. Then,

$$
|(A u, v)| \leq \max _{j=k, \cdots, n}\left|\lambda_{j}\right| \sum_{j=k}^{n}\left(u, u_{j}^{*}\right)\left(p_{j}, v\right)=\left|\lambda_{k}\right|(u, v),
$$

that is,

$$
\frac{|(A u, v)|}{(u, v)} \leq\left|\lambda_{k}(A)\right|
$$

and thus

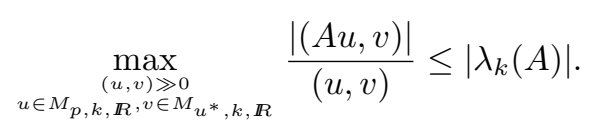

Now, $\left|\lambda_{k}(A)\right|$ is attained for $u=p_{k} \in M_{p, k, \mathbb{R}}$ and $v \in M_{u^{*}, k, \mathbb{R}}$, that is,

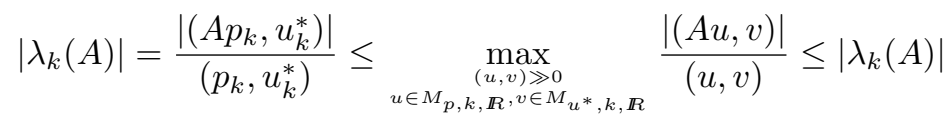

so that (67) is proven. The last assertion is clear.

Remark: It seems that min-, maxmin-, and minmax-representations cannot be derived. At least, it is not clear to the author how this could be done. The reason for a minrepresentation is as follows. In the proof of of Theorem 13, one has

$$
|(A u, v)| \nsupseteq \sum_{j=1}^{k}\left|\lambda_{j}\right|\left(u, u_{j}^{*}\right)\left(p_{j}, v\right),(u, v) \gg 0, u \in N_{p, k, \mathbb{R}}, v \in N_{u^{*}, k, \mathbb{R}}
$$

The next way is neither successful. One has

$$
\begin{aligned}
\operatorname{Re}(A u, v) & \geq \min _{j=1 \cdots, k} \operatorname{Re} \lambda_{j}(u, v), \\
\operatorname{Im}(A u, v) & \geq \min _{j=1 \cdots, k} \operatorname{Im} \lambda_{j}(u, v),
\end{aligned}
$$

$(u, v) \gg 0, u \in N_{p, k, \mathbb{R}}, v \in N_{u^{*}, k, \mathbb{R}}$ leading to

$$
\begin{aligned}
|(A u, v)|^{2} & \geq\left[\left(\min _{j=1 \cdots, k} \operatorname{Re} \lambda_{j}\right)^{2}+\left(\min _{j=1 \cdots, k} \operatorname{Im} \lambda_{j}\right)^{2}\right](u, v)^{2} \\
& \geq \min _{j=1 \cdots, k}\left[\left(\operatorname{Re} \lambda_{j}\right)^{2}+\left(\operatorname{Im} \lambda_{j}\right)^{2}\right](u, v)^{2} \\
& =\min _{j=1 \cdots, k}\left|\lambda_{j}\right|^{2}(u, v)^{2}=\left(\min _{j=1 \cdots, k}\left|\lambda_{j}\right|\right)^{2}(u, v)^{2} .
\end{aligned}
$$

Remark: In the Appendix, we show that, with a minor additional hypothesis, the min-, minmax-, and maxmin-representations can be proven.

\section{Generalized Rayleigh-quotient formulas for a diagonalizable matrix with real eigenvalues}

In Section 4, we have observed that, for the moduli of the eigenvalues of a diagonalizable matrix, one obtains only a max-representation with generalized Rayleigh quotients. However, for $A \in \mathbb{C}^{n \times n}$ with

$$
\sigma(A) \subset \mathbb{R},
$$


one gets generalized Rayleigh-quotient formulas for the eigenvalues themselves. And it goes without saying that these imply Rayleigh-quotient representations for the moduli if all eigenvalues are nonnegative such as $\lambda_{1}\left(A^{*} A\right), \cdots, \lambda_{n}\left(A^{*} A\right)$.

So, let $A \in \mathbb{C}^{n \times n}$ with spectrum $\sigma(A) \subset \mathbb{R}$. Further, let the eigenvalues be arranged according to

$$
\lambda_{1}(A) \geq \lambda_{2}(A) \geq \cdots \geq \lambda_{n}(A) .
$$

Then, we obtain the following series of corollaries following from Theorems $4-7$, as the case may be.

\section{Corollary 14:}

Let the conditions $(C 1)-(C 4)$ be fulfilled. Further, let $\sigma(A) \subset \mathbb{R}$, and let the eigenvalues of $A$ be arranged according to (70). Moreover, let the vector spaces $M_{p, k, \mathbb{R}}$ and $M_{u^{*}, k, \mathbb{R}}$ for $k=1, \cdots, n$ be defined by (16), (17) or (18),(19).

Then,

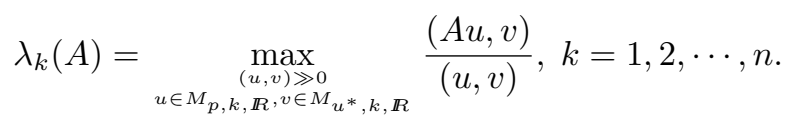

The maximum is attained for $u=p_{k}, v=u_{k}^{*}$.

\section{Corollary 15:}

Let the conditions $(C 1)-(C 4)$ be fulfilled. Further, let $\sigma(A) \subset \mathbb{R}$, and let the eigenvalues of $A$ be arranged according to (70).

Then, for every $j=1, \cdots, n$ and every subspace $M_{p} \subset N_{p, \mathbb{R}}$ and $M_{u^{*}} \subset N_{u^{*}, \mathbb{R}}$ with $\operatorname{dim} M_{p}=\operatorname{dim} M_{u^{*}}=m=n+1-j$, the following inequalities are valid:

$$
\lambda_{j}(A) \leq \max _{\substack{(u, v) \gg 0 \\ u \in M_{p}, v \in M_{u^{*}}}} \frac{(A u, v)}{(u, v)} \leq \lambda_{1}(A),
$$

and the following representation formulas hold:

$$
\lambda_{j}(A)=\min _{\substack{\operatorname{dim} M_{p}=m \\ \operatorname{dim} M_{u^{*}=m}}} \max _{\substack{(u, v) \gg 0 \\ u \in M_{p}, v \in M_{u^{*}}}} \frac{(A u, v)}{(u, v)} .
$$

Remark: From (72), it follows

$$
\frac{(A u, v)}{(u, v)} \leq \max _{j=1, \cdots, n} \lambda_{j}(A),(u, v) \gg 0, u \in N_{p, \mathbb{R}}, v \in N_{u^{*}, \mathbb{R}} .
$$

\section{Corollary 16:}

Let the conditions $(C 1)-(C 4)$ be fulfilled. Further, let $\sigma(A) \subset \mathbb{R}$, and let the eigenvalues of $A$ be arranged according to (70). Moreover, let the vector spaces $N_{p, k, \mathbb{R}}$ and $N_{u^{*}, k, \mathbb{R}}$ for $k=1, \cdots, n$ be defined by (3) and (7).

Then,

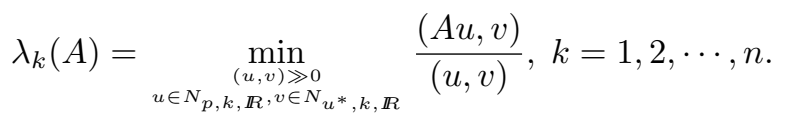

The minimum is attained for $u=p_{k}, v=u_{k}^{*}$. 


\section{Corollary 17:}

Let the conditions $(C 1)-(C 4)$ be fulfilled. Further, let $\sigma(A) \subset \mathbb{R}$, and let the eigenvalues of $A$ be arranged according to (70).

Then, for every $j=1, \cdots, n$ and all subspaces $N_{p} \subset N_{p, \mathbb{R}}$ and $N_{u^{*}} \subset N_{u^{*}, \mathbb{R}}$ with $\operatorname{dim} N_{p}=\operatorname{dim} N_{u^{*}}=j$, the following inequalities are valid:

$$
\lambda_{n}(A) \leq \min _{\substack{u, v)>0 \\ u \in N_{p}, v \in N_{u^{*}}}} \frac{(A u, v)}{(u, v)} \leq \lambda_{j}(A),
$$

and the following representation formulas hold:

$$
\lambda_{j}(A)=\max _{\substack{\operatorname{dim} N_{p}=j \\ \operatorname{dim} N_{u^{*}}=j}} \min _{\substack{(u, v)>0 \\ u \in N_{p}, v \in N_{u^{*}}}} \frac{(A u, v)}{(u, v)} .
$$

Remark: From (75), it follows

$$
\frac{(A u, v)}{(u, v)} \geq \min _{j=1, \cdots, n} \lambda_{j}(A),(u, v) \gg 0, u \in N_{p, \mathbb{R}}, v \in N_{u^{*}, \mathbb{R}} .
$$

\section{Application}

In this section, an application of the obtained results is presented. More precisely, a new formula for $\rho(A)$ is derived. First, known formulas for this quantity are recapitulated.

Known formulas for the spectral radius of $A \in \mathbb{C}^{n \times n}$

One formula is given by

$$
\rho(A)=\lim _{n \rightarrow \infty}\left\|A^{n}\right\|^{\frac{1}{n}},
$$

see [4, Chapter I, p.27], where in (77) the spectral radius $\rho(A)$ is independent of the used submultiplicative norm $\|\cdot\|$.

Another representation is

$$
\rho(A)=\max _{j=1, \cdots, n}\left|\lambda_{j}(A)\right|,
$$

cf. [4, Chapter I,(5.12), p.38].

New formula for the spectral radius of $A \in \mathbb{C}^{n \times n}$

Let the conditions $(C 1)$ - $(C 4)$ be fulfilled. Then, from Theorem 13, as Application, we deduce the new formula

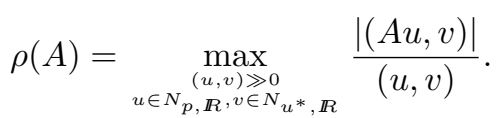

Proof: This follows from (67) with the arrangement (66) with $k=1$ as well as $M_{p, 1, \mathbb{R}}=N_{p, \mathbb{R}}$ and $M_{u^{*}, 1, \mathbb{R}}=N_{u^{*}, \mathbb{R}}$ according to (18), (19).

\section{New generalized numerical ranges}

In this section, a series of known numerical ranges are recapitulated and new numerical ranges of a matrix are defined. 
Known numerical range of $A \in \mathbb{C}^{n \times n}$ with respect to the full space $\mathbb{C}^{n}$

According to $\left[9\right.$, Section 5.4,(5)], the numerical range of $A \in \mathbb{C}^{n \times n}$ with respect to the full space $\mathbb{C}^{n}$ is defined by

$$
W_{\mathbb{C}^{n}}(A)=\left\{z \in \mathbb{C} \mid z=\frac{(A u, u)}{(u, u)}, 0 \neq u \in \mathbb{C}^{n}\right\},
$$

which is a convex subset of $\mathbb{C}$. Employing this definition to $A^{*} A$ instead of $A$, we obtain

$$
W_{\mathbb{C}^{n}}\left(A^{*} A\right)=\left\{x \in \mathbb{R}_{0}^{+} \mid x=\frac{\left(A^{*} A u, u\right)}{(u, u)}=\frac{(A u, A u)}{(u, u)}, 0 \neq u \in \mathbb{C}^{n}\right\},
$$

which is a convex subset of $\mathbb{R}_{0}^{+}$. One has

$$
W_{\mathbb{C}^{n}}\left(A^{*} A\right)=\left[\min _{j=1, \cdots, n} \lambda_{j}\left(A^{*} A\right), \max _{j=1, \cdots, n} \lambda_{j}\left(A^{*} A\right)\right]=\left[\frac{1}{\left\|A^{-1}\right\|_{2}^{2}},\|A\|_{2}^{2}\right]
$$

where $\frac{1}{\left\|A^{-1}\right\|_{2}^{2}}$ has to be interpreted as zero if $A$ is singular.

$\underline{\text { Known generalized numerical range for self-adjoint } A \in \mathbb{C}^{n \times n} \text { with respect to the }}$ subspace $N_{\mathbb{R}}$

For self-adjoint $A \in \mathbb{C}^{n \times n}$, we define the generalized numerical range with respect to the subspace $N_{\mathbb{R}}=\left[w_{1}, \cdots, w_{n}\right]_{\mathbb{R}}$ with the eigenvectors $w_{i}=w_{i}(A), i=1, \cdots, n$ by

$$
W_{N_{\mathbb{R}}, \text { generalized }}(A)=\left\{x \in \mathbb{R} \mid x=\frac{(A u, v)}{(u, v)},(u, v) \gg 0, u, v \in N_{\mathbb{R}}\right\} .
$$

Employing this definition to $A^{*} A$ instead of general $A \in \mathbb{C}^{n \times n}$, we obtain

$$
W_{N_{\mathbb{R}}, \text { generalized }}\left(A^{*} A\right)=\left\{x \in \mathbb{R} \mid x=\frac{\left(A^{*} A u, v\right)}{(u, v)}=\frac{(A u, A v)}{(u, v)},(u, v) \gg 0, u, v \in N_{\mathbb{R}}\right\}
$$

where $N_{\mathbb{R}}=\left[w_{1}, \cdots, w_{n}\right]_{\mathbb{R}}$ with the eigenvectors $w_{i}=w_{i}\left(A^{*} A\right), i=1, \cdots, n$. Here,

$$
W_{N_{\mathbb{R}}, \text { generalized }}\left(A^{*} A\right)=W_{\mathbb{C}^{n}}\left(A^{*} A\right)
$$

since

$$
W_{N_{\mathbb{R}}, \text { generalized }}\left(A^{*} A\right)=\left[\min _{j=1, \cdots, n} \lambda_{j}\left(A^{*} A\right), \max _{j=1, \cdots, n} \lambda_{j}\left(A^{*} A\right)\right]
$$

the proof of which is left to the reader.

The following four definitions of generalized numerical ranges are new.

$\underline{\text { Generalized numerical range of } A \in \mathbb{C}^{n \times n} \text { with respect to the subspaces } N_{p, \mathbb{R}}}$ and $N_{u^{*}, \mathbb{R}}$

Let the conditions $(C 1)$ - $(C 4)$ be fulfilled. Then, we define the generalized numerical range of $A$ with respect to the subspaces $N_{p, \mathbb{R}}$ and $N_{u^{*}, \mathbb{R}}$ by

$$
W_{N_{p, \mathbb{R}}, N_{u^{*}, \mathbb{R}}, g e n .}(A)=\left\{z \in \mathbb{C} \mid z=\frac{(A u, v)}{(u, v)},(u, v) \gg 0, u \in N_{p, \mathbb{R}}, v \in N_{u^{*}, \mathbb{R}}\right\} .
$$

Real part of the generalized numerical range of $A \in \mathbb{C}^{n \times n}$ with respect to the subspaces $N_{p, \mathbb{R}}$ and $N_{u^{*}, \mathbb{R}}$ 
Let the conditions $(C 1)-(C 4)$ be fulfilled. Then, we define the real part of the generalized numerical range of $A$ with respect to the subspaces $N_{p, \mathbb{R}}$ and $N_{u^{*}, \mathbb{R}}$ by

$\operatorname{Re}\left[W_{N_{p, \mathbb{R}}, N_{u^{*}, \mathbb{R}}, g e n .}(A)\right]=\left\{x \in \mathbb{R} \mid x=\frac{\operatorname{Re}(A u, v)}{(u, v)},(u, v) \gg 0, u \in N_{p, \mathbb{R}}, v \in N_{u^{*}, \mathbb{R}}\right\}$.

Imaginary part of the generalized numerical range of $A \in \mathbb{C}^{n \times n}$ with respect to the subspaces $N_{p, \mathbb{R}}$ and $N_{u^{*}, \mathbb{R}}$

Let the conditions $(C 1)-(C 4)$ be fulfilled. Then, we define the imaginary part of the generalized numerical range of $A$ with respect to the subspaces $N_{p, \mathbb{R}}$ and $N_{u^{*}, \mathbb{R}}$ by

$\operatorname{Im}\left[W_{N_{p, \mathbb{R}}, N_{u^{*}, \mathbb{R}}, g e n .}(A)\right]=\left\{x \in \mathbb{R} \mid x=\frac{\operatorname{Im}(A u, v)}{(u, v)},(u, v) \gg 0, u \in N_{p, \mathbb{R}}, v \in N_{u^{*}, \mathbb{R}}\right\}$.

Modulus of the generalized numerical range of $A \in \mathbb{C}^{n \times n}$ with respect to

the subspaces $N_{p, \mathbb{R}}$ and $N_{u^{*}, \mathbb{R}}$

Let the conditions $(C 1)$ - $(C 4)$ be fulfilled. Then, we define the modulus of the generalized numerical range of $A$ with respect to the subspaces $N_{p, \mathbb{R}}$ and $N_{u^{*}, \mathbb{R}}$ by

$$
\mid\left[W_{N_{p, \mathbb{R}}, N_{u^{*}, \mathbb{R}}, g e n .}(A) \mid=\left\{x \in \mathbb{R}_{0}^{+} \mid x=\frac{|(A u, v)|}{(u, v)},(u, v) \gg 0, u \in N_{p, \mathbb{R}}, v \in N_{u^{*}, \mathbb{R}}\right\} .\right.
$$

\section{Numerical Example 1}

In this section, we check some of the formulas of Section 2 on an example from the theory of linear dynamical systems. More precisely, we check the validity of the following relation

$$
\frac{\operatorname{Re}(A u, v)}{(u, v)} \in\left[\min _{j=1, \cdots, n} \operatorname{Re} \lambda_{j}(A), \max _{j=1, \cdots, n} \operatorname{Re} \lambda_{j}(A)\right],
$$

$(u, v) \gg 0, u \in N_{p, \mathbb{R}}, v \in N_{u^{*}, \mathbb{R}}$ for a series of vectors $u \in N_{p, \mathbb{R}}$ and $N_{u^{*}, \mathbb{R}}$ that is a consequence of Theorems 4 and 6 .

\subsection{A multi-mass vibration model}

We take up the multi-mass vibration model of [7], shown in Fig.1.

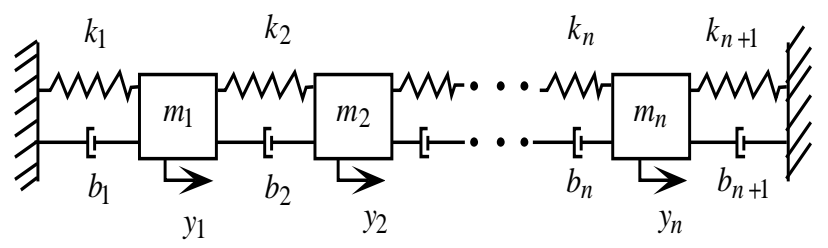

Fig.1: Multi-mass vibration model

The associated initial value problem is given by

$$
M \ddot{y}+B \dot{y}+K y=0, y(0)=y_{0}, \dot{y}(0)=\dot{y}_{0},
$$

where $y=\left[y_{1}, \cdots, y_{n}\right]^{T}$ and

$$
M=\left[\begin{array}{lllll}
m_{1} & & & & \\
& m_{2} & & & \\
& & m_{3} & & \\
& & & \ddots & \\
& & & & m_{n}
\end{array}\right],
$$




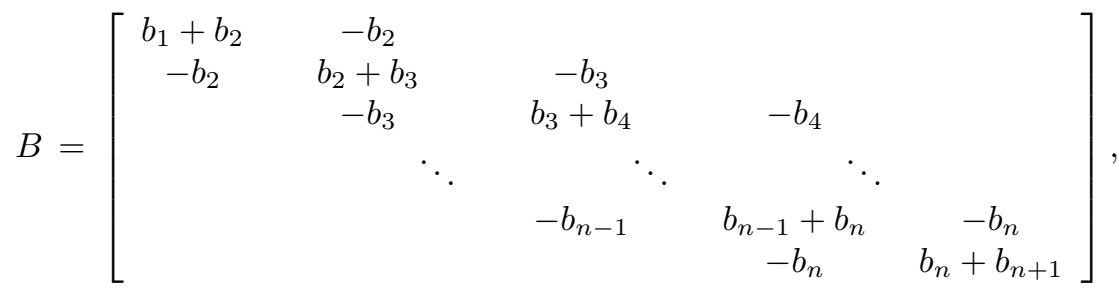

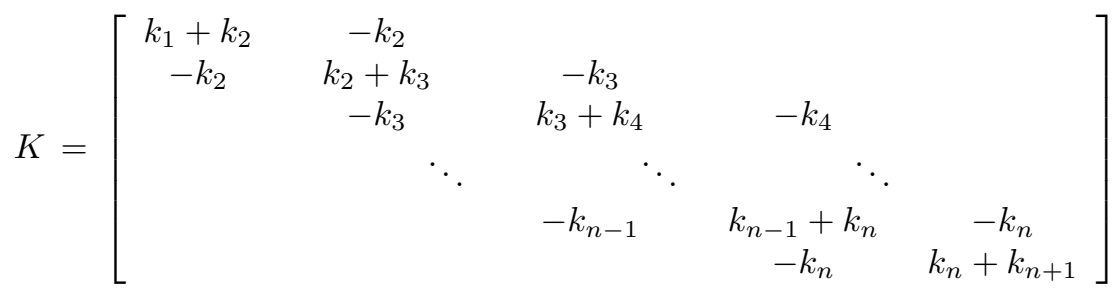

with the mass, damping, and stiffness matrices $M, B$, and $K$, as the case may be, and the displacement vector $y$ as in [7]. In state-space description, this problem takes the form

$$
\dot{x}=A x, t \geq 0, x(0)=x_{0},
$$

where $x=\left[y^{T}, z^{T}\right]^{T}, z=\dot{y}$, and where the system matrix $A$ is given by

$$
A=\left[\begin{array}{c|c}
0 & E \\
\hline-M^{-1} K & -M^{-1} B
\end{array}\right] .
$$

\subsection{Data}

The values $m_{j}, j=1, \cdots, n$ and $b_{j}, k_{j}, j=1, \cdots, n+1$ are also specified as in [7], namely as

$$
\begin{aligned}
m_{j} & =1, j=1, \cdots, n \\
k_{j} & =1, j=1, \cdots, n+1 \\
\text { and } & \\
b_{j} & =\left\{\begin{array}{l}
1 / 2, j \text { even } \\
1 / 4, j \text { odd } .
\end{array}\right.
\end{aligned}
$$

Then,

$$
B=\left[\begin{array}{rrrrr}
\frac{3}{4} & -\frac{1}{2} & & & \\
-\frac{1}{2} & \frac{3}{4} & -\frac{1}{4} & & \\
& -\frac{1}{4} & \frac{3}{4} & -\frac{1}{2} & \\
& & \ddots & \ddots & \ddots \\
& & -\frac{1}{4} & \frac{3}{4} & -\frac{1}{2} \\
& & & -\frac{1}{2} & \frac{3}{4}
\end{array}\right]
$$

(if $n$ is even), and

$$
K=\left[\begin{array}{rrrrr}
2 & -1 & & & \\
-1 & 2 & -1 & & \\
& -1 & 2 & -1 & \\
& & \ddots & \ddots & \ddots \\
& & -1 & 2 & -1 \\
& & & -1 & 2
\end{array}\right]
$$


We add the details from [7] in order to make the paper more readable on its own. Further, we choose $n=5$ in this paper so that the state-space vector has dimension $m=2 n=10$. For the initial time, we take

$$
t_{0}=0 \text {. }
$$

Finally, the initial conditions for $y(t)$ and $\dot{y}(t)$ can be chosen as

$$
y_{0}=[-1,1,-1,1,-1]^{T}
$$

as well as

$$
\dot{y}_{0}=[-1,-1,-1,-1,-1]^{T} .
$$

But, they are not needed here.

\subsection{Auxiliary computational results}

Using the Matlab routine eig.m, for the eigenvalues, one obtains

\begin{tabular}{r|r|r}
$i$ & $\lambda_{i}=\lambda_{i}(A)$ & $\bar{\lambda}_{i}=\lambda_{i}\left(A^{*}\right)$ \\
\hline 1 & $-0.699760638780535+1.795981478159750 i$ & $-0.699760638780535-1.795981478159750 i$ \\
2 & $-0.562668374040743+1.616358701643862 i$ & $-0.562668374040743-1.616358701643862 i$ \\
3 & $-0.375000000000000+1.363589014329463 i$ & $-0.375000000000000-1.363589014329463 i$ \\
4 & $-0.187331625959257+0.994521686465592 i$ & $-0.187331625959257-0.994521686465592 i$ \\
5 & $-0.050239361219464+0.516371450711011 i$ & $-0.050239361219464-0.516371450711011 i$
\end{tabular}

and

$$
\lambda_{5+i}=\bar{\lambda}_{i}, i=1, \cdots, 5
$$

where the numbering is chosen such that $\operatorname{Im} \lambda_{i}>0, i=1, \cdots, 5$. So,

$$
\lambda_{i} \neq \lambda_{j}, i \neq j, i, j=1, \cdots, 10
$$

Therefore, $\lambda_{j}(A), j=1, \cdots, m=2 n=10$ are distinct. Thus, matrix $A$ is diagonalizable, regular and asymptotically stable. The eigenvalues are ordered such that $\operatorname{Re} \lambda_{1}(A) \geq \cdots \geq \operatorname{Re} \lambda_{5}(A)$. We mention that we need not the arrangement (21), here. Further, we use only 6 digits for space reasons; all calculations were made with full precision, however. We obtain

$$
\left.\begin{array}{r}
p_{1}=\left[\begin{array}{r}
-0.024551-0.145308 i \\
0.081481+0.213285 i \\
-0.096403-0.247425 i \\
0.081481+0.213285 i \\
-0.071853-0.102117 i \\
0.278150+0.057589 i \\
-0.440072-0.002910 i \\
0.511830 \\
-0.440072-0.002910 i \\
0.233679-0.057589 i
\end{array}\right], \quad u_{1}^{*}=\left[\begin{array}{r}
-0.038673-0.357284 i \\
0.016641+0.598392 i \\
0.004834-0.681876 i \\
-0.024449+0.582046 i \\
0.043506-0.324592 i \\
0.165567-0.105085 i \\
-0.283480+0.109299 i \\
0.330529-0.124935 i \\
-0.283480+0.109299 i \\
0.164963-0.019850 i \\
0.089443+0.256940 i \\
-0.108651-0.210030 i \\
0.071526-0.080434 i \\
0.108651+0.210030 i \\
-0.160968-0.176505 i \\
-0.465633 \\
0.400619-0.057442 i \\
0.089766+0.160869 i \\
-0.400619+0.057442 i \\
0.375868-0.160869 i
\end{array}\right], \\
p_{2} \\
0.157251+0.597292 i \\
-0.039411-0.558737 i \\
-0.167005-0.035323 i \\
0.122913+0.576399 i \\
0.009755-0.561969 i \\
-0.297136+0.219170 i \\
0.282686-0.151912 i \\
-0.018437-0.144908 i \\
-0.282686+0.151912 i \\
0.315573-0.074262 i
\end{array}\right],
$$




$$
p_{3}=\left[\begin{array}{r}
-0.245285-0.177230 i \\
-0.000000+0.000000 i \\
0.189016+0.272329 i \\
0.000000-0.000000 i \\
-0.096291-0.350137 i \\
0.333651-0.268016 i \\
-0.000000-0.000000 i \\
-0.442226+0.155617 i \\
0.000000+0.000000 i \\
0.513553
\end{array}\right],
$$$$
p_{4}=\left[\begin{array}{r}
-0.067070-0.356067 i \\
-0.100458-0.328255 i \\
-0.077742+0.042653 i \\
0.100458+0.328255 i \\
0.144812+0.313414 i \\
0.366681 \\
0.345275-0.038415 i \\
-0.027856-0.085307 i \\
-0.345275+0.038415 i \\
-0.338825+0.085307 i
\end{array}\right] \text {, }
$$$$
p_{5}=\left[\begin{array}{r}
0.257864+0.016537 i \\
0.443516+0.000061 i \\
0.512481 \\
0.443516+0.000061 i \\
0.254617-0.016537 i \\
-0.021494+0.132323 i \\
-0.022313+0.229016 i \\
-0.025747+0.264631 i \\
-0.022313+0.229016 i \\
-0.004253+0.132308 i
\end{array}\right],
$$

$$
u_{3}^{*}=\left[\begin{array}{r}
0.048677-0.548046 i \\
-0.098830-0.017984 i \\
0.148982+0.584014 i \\
0.108664-0.017353 i \\
-0.366310-0.549308 i \\
0.382782-0.069571 i \\
-0.000000+0.000000 i \\
-0.370244+0.211078 i \\
0.000000+0.000000 i \\
0.305832-0.352743 i
\end{array}\right],
$$$$
u_{4}^{*}=\left[\begin{array}{r}
-0.127071-0.395332 i \\
-0.087749-0.364203 i \\
0.190090+0.019705 i \\
-0.007296+0.354350 i \\
-0.063019+0.375627 i \\
0.364166-0.153330 i \\
0.358971-0.106228 i \\
0.008007+0.096370 i \\
-0.358971+0.106228 i \\
-0.372173+0.056960 i
\end{array}\right],
$$

$$
u_{5}^{*}=\left[\begin{array}{r}
0.178929-0.061419 i \\
0.272848+0.066164 i \\
0.327018+0.032486 i \\
0.288268-0.011499 i \\
0.148089+0.093905 i \\
0.020074+0.318770 i \\
-0.000556+0.548232 i \\
-0.000729+0.633480 i \\
-0.000556+0.548232 i \\
-0.020803+0.314709 i
\end{array}\right]
$$

where $p_{i}=p_{i}(A), u_{i}^{*}=u_{i}^{*}\left(A^{*}\right) \in \mathbb{R}^{10}, i=1, \cdots, 5$ and $p_{5+i}=\overline{p_{i}}, u_{5+i}^{*}=\overline{u_{i}^{*}}, i=$ $1, \cdots, 5$ with the property $\left(p_{i}, u_{j}^{*}\right)=\delta_{i j}, i, j=1, \cdots, 10$.

Remark: The pairs of orthonormal eigenvectors $p_{i}=p_{i}(A), u_{i}^{*}=u_{i}^{*}\left(A^{*}\right), i=1, \cdots, 5$ have already been computed in [6]. But, the results for the right eigenvectors look very different. Here, the computations were done by employing Matlab, Version 7.11, those in [6] by Matlab, Version 4.2c. For instance, in [6], we have

$$
p_{3,[6]}=\left[\begin{array}{r}
-0.003137-0.302598 i \\
0.000000+0.000000 i \\
-0.106791+0.313825 i \\
0.000000-0.000000 i \\
0.224464-0.285454 i \\
0.413795+0.109197 i \\
0.000000+0.000000 i \\
-0.387881-0.263303 i \\
0.000000+0.000000 i \\
0.305068+0.413122 i
\end{array}\right] \text {, }
$$$$
u_{3,[6]}^{*}=\left[\begin{array}{r}
0.469786-0.286400 i \\
-0.044241-0.090186 i \\
-0.381304+0.466772 i \\
0.078510+0.077105 i \\
0.224285-0.620982 i \\
0.283351+0.266598 i \\
0.000000-0.000000 i \\
-0.389737-0.172451 i \\
0.000000+0.000000 i \\
0.465435+0.036482 i
\end{array}\right] .
$$ 
The reason for the different outcomes is as follows. For any system $p_{i}, u_{i}^{*}, i=1, \cdots, 5$ with $\left(p_{i}, u_{j}^{*}\right)=\delta_{i j}, i, j=1, \cdots, 5$, also the system $p_{i} e^{i \varphi}, u_{i}^{*} e^{i \varphi}, i=1, \cdots, 5$ satisfies $\left(p_{i} e^{i \varphi}, u_{j}^{*} e^{i \varphi}\right)=\delta_{i j}, i, j=1, \cdots, 5$ for $0 \leq \varphi \leq 2 \pi$. For example, here, we obtain $p_{3,[6]}=p_{3} e^{i \varphi_{3}}$ and $u_{3,[6]}^{*}=u_{3}^{*} e^{i \varphi_{3}}$ with $\varphi_{3}=0.934732250297$.

\subsection{Numerical check of Theorems 4 and 6}

From Theorem 4, Formula (22) and Theorem 6, Formula (41), we conclude

$$
\min _{j=1, \cdots, 10} \operatorname{Re} \lambda_{j}(A) \leq \frac{\operatorname{Re}(A u, v)}{(u, v)} \leq \max _{j=1, \cdots, 10} \operatorname{Re} \lambda_{j}(A)
$$

$(u, v) \gg 0, u \in N_{p, \mathbb{R}}, v \in N_{u^{*}, \mathbb{R}}$ by setting $k=1$, there. This can also be written as

$$
\frac{\operatorname{Re}(A u, v)}{(u, v)} \in\left[\min _{j=1, \cdots, 10} \operatorname{Re} \lambda_{j}(A), \max _{j=1, \cdots, 10} \operatorname{Re} \lambda_{j}(A)\right]
$$

$(u, v) \gg 0, u \in N_{p, \mathbb{R}}, v \in N_{u^{*}, \mathbb{R}}$. We check this for a series of vectors. One has $\left[\min _{j=1, \cdots, 10} \operatorname{Re} \lambda_{j}(A), \max _{j=1, \cdots, 10} \operatorname{Re} \lambda_{j}(A)\right]=[-0.699760638780535,-0.050239361219464]$.

Let

$$
\begin{aligned}
& u_{1}=-5 p_{4}+3 p_{10}, \\
& v_{1}=-4 u_{4}^{*}+2 u_{10}^{*} .
\end{aligned}
$$

Then $u_{1} \in N_{p, \mathbb{R}}$ and $v_{1} \in N_{u^{*}, \mathbb{R}}$ as well as $\left(u_{1}, v_{1}\right) \gg 0$, and one obtains

$$
\begin{gathered}
{\left[\begin{array}{r}
1.108943+1.730727 i \\
1.832839+1.641092 i \\
1.926154-0.213267 i \\
0.828258-1.641455 i \\
0.039789-1.517460 i \\
-1.897887-0.396968 i \\
-1.793317-0.494972 i \\
0.062040-0.367358 i \\
1.659438-0.879125 i \\
1.681366-0.823456 i
\end{array}\right], \quad v_{1}=\left[\begin{array}{r}
0.866142+1.704164 i \\
0.896690+1.324484 i \\
-0.106323-0.143792 i \\
0.605720-1.394405 i \\
0.548252-1.690318 i \\
-1.416517-0.024220 i \\
-1.436997-0.671555 i \\
-0.033485-1.652439 i \\
1.434772-1.521375 i \\
1.447085-0.857261 i
\end{array}\right],} \\
\left(A u_{1}, v_{1}\right)=-4.048068686501926+16.792205025045781 i, \\
\left(u_{1}, v_{1}\right)=25.999999999999989+0.000000000000001 i \approx 26,
\end{gathered}
$$

and thus

$$
\frac{\operatorname{Re}\left(A u_{1}, v_{1}\right)}{\left(u_{1}, v_{1}\right)} \doteq-0.155694949480843 \in\left[\min _{j=1, \cdots, 10} \operatorname{Re} \lambda_{j}(A), \max _{j=1, \cdots, 10} \operatorname{Re} \lambda_{j}(A)\right] .
$$

Let

$$
\begin{array}{rrr}
u_{2}= & 3 p_{10}, \\
v_{2}= & -4 u_{4}^{*}+2 u_{10}^{*} .
\end{array}
$$

Then $u_{2} \in N_{p, \mathbb{R}}$ and $v_{2} \in N_{u^{*}, \mathbb{R}}$ as well as $\left(u_{2}, v_{2}\right) \gg 0$, and one obtains

$$
u_{2}=\left[\begin{array}{r}
0.773592-0.049610 i \\
1.330549-0.000182 i \\
1.537443 \\
1.330549-0.000182 i \\
0.763851+0.049610 i \\
-0.064482-0.396968 i \\
-0.066940-0.687048 i \\
-0.077240-0.793892 i \\
-0.066940-0.687048 i \\
-0.0127583-0.396923 i
\end{array}\right]
$$$$
v_{2}=\left[\begin{array}{r}
0.866142+1.704164 i \\
0.896690+1.324484 i \\
-0.106323-0.143792 i \\
0.605720-1.394405 i \\
0.548252-1.690318 i \\
-1.416517-0.024220 i \\
-1.436997-0.671555 i \\
-0.033485-1.652439 i \\
1.434772-1.521375 i \\
1.447085-0.857261 i
\end{array}\right],
$$ 


$$
\begin{aligned}
\left(A u_{2}, v_{2}\right) & =-0.301436167316785-3.098228704266065 i \\
\left(u_{2}, v_{2}\right) & =5.999999999999991+0.000000000000004 i \approx 6,
\end{aligned}
$$

and thus

$$
\frac{\operatorname{Re}\left(A u_{2}, v_{2}\right)}{\left(u_{2}, v_{2}\right)} \doteq-0.050239361219464 \in\left[\min _{j=1, \cdots, 10} \operatorname{Re} \lambda_{j}(A), \max _{j=1, \cdots, 10} \operatorname{Re} \lambda_{j}(A)\right] .
$$

Let

$$
\begin{aligned}
& u_{3}=-5 p_{1}+3 p_{8}, \\
& v_{3}=-4 u_{1}^{*}+2 u_{8}^{*} .
\end{aligned}
$$

Then $u_{3} \in N_{p, \mathbb{R}}$ and $v_{3} \in N_{u^{*}, \mathbb{R}}$ as well as $\left(u_{3}, v_{3}\right) \gg 0$, and one obtains

$$
\begin{gathered}
{\left[\begin{array}{r}
-0.613102+1.258231 i \\
-0.407405-1.066423 i \\
1.049063+0.420138 i \\
-0.407405-1.066423 i \\
0.070390+1.560996 i \\
-0.389800+0.516076 i \\
2.200362+0.014549 i \\
-3.885826-0.466850 i \\
2.200362+0.014549 i \\
0.372261+0.287943 i
\end{array}\right], \quad v_{3}=\left[\begin{array}{r}
0.252046+2.525227 i \\
-0.264224-2.357598 i \\
0.278630+1.559474 i \\
0.315122-2.293476 i \\
-0.906646+2.396983 i \\
0.103296+0.559481 i \\
1.133919-0.437195 i \\
-2.062605+0.077585 i \\
1.133919-0.437195 i \\
-0.048187+0.784889 i
\end{array}\right],} \\
\left(A u_{3}, v_{3}\right)=-16.245212775610707+27.738095477218263 i, \\
\left(u_{3}, v_{3}\right)=26.000000000000004-0.000000000000000 i \approx 26,
\end{gathered}
$$

and thus

$$
\frac{\operatorname{Re}\left(A u_{3}, v_{3}\right)}{\left(u_{3}, v_{3}\right)} \doteq-0.624815875985027 \in\left[\min _{j=1, \cdots, 10} \operatorname{Re} \lambda_{j}(A), \max _{j=1, \cdots, 10} \operatorname{Re} \lambda_{j}(A)\right] .
$$

Let

$$
\begin{array}{rrr}
u_{4}= & -5 p_{9}+3 p_{10}, \\
v_{4}= & -2 u_{8}^{*} .
\end{array}
$$

Then $u_{4} \in N_{p, \mathbb{R}}$ and $v_{4} \in N_{u^{*}, \mathbb{R}}$ as well as $\left(u_{4}, v_{4}\right) \ngtr 0$, and one obtains

$$
\begin{array}{r}
u_{4}=\left[\begin{array}{r}
1.108942-1.829946 i \\
1.832839-1.641455 i \\
1.926154+0.213267 i \\
0.828258+1.641092 i \\
0.039789+1.616680 i \\
-1.897887-0.396968 i \\
-1.793317-0.879125 i \\
0.062040-1.220425 i \\
1.659438-0.494972 i \\
1.681366+0.029610 i
\end{array}\right], \quad v_{4}=\left[\begin{array}{r}
-0.097355-1.096092 i \\
0.197660-0.035968 i \\
-0.297965+1.168029 i \\
-0.217328-0.034706 i \\
0.732620-1.098616 i \\
-0.765563-0.139141 i \\
0.000000+0.000000 i \\
0.740487+0.422156 i \\
-0.000000+0.000000 i \\
-0.611664-0.705487 i
\end{array}\right], \\
\left(A u_{4}, v_{4}\right)=-2.731148640577885 \times 10^{-14}-6.661338147750939 \times 10^{-15} i, \\
\left(u_{4}, v_{4}\right)=1.132427485117660 \times 10^{-14}-1.754152378907747 \times 10^{-14} i \approx 0,
\end{array}
$$

and thus

$$
\begin{aligned}
\frac{\operatorname{Re}\left(A u, v_{4}\right)}{\left(u_{4}, v_{4}\right)} & =-0.709454874462791-1.098959511422755 i \\
& \notin\left[\min _{j=1, \cdots, 10} \operatorname{Re} \lambda_{j}(A), \max _{j=1, \cdots, 10} \operatorname{Re} \lambda_{j}(A)\right]
\end{aligned}
$$

which is not surprising since $\left(u_{4}, v_{4}\right) \approx 0$. 
Let

$$
\begin{aligned}
& u_{5}=[1,2,3,4,5,6,7,8,9,10]^{T} \in \mathbb{R}^{10} \\
& v_{5}=[10,9,8,7,6,5,4,3,2,1]^{T} \in \mathbb{R}^{10}
\end{aligned}
$$

Here, one obtains

$$
\begin{gathered}
\left(A u_{5}, v_{5}\right)=293 \\
\left(u_{5}, v_{5}\right)=220
\end{gathered}
$$

and thus

$$
\frac{\operatorname{Re}\left(A u_{5}, v_{5}\right)}{\left(u_{5}, v_{5}\right)}=1.331818181818182 \notin\left[\min _{j=1, \cdots, 10} \operatorname{Re} \lambda_{j}(A), \max _{j=1, \cdots, 10} \operatorname{Re} \lambda_{j}(A)\right]
$$

which is neither surprising since $\left(u_{5}, v_{5}\right) \ngtr 0$ due to

$$
\alpha^{(5)}:=\left(\alpha_{k}^{(5)}\right)_{k=1, \cdots, 10}=\left(\left(u_{5}, u_{k}^{*}\right)\right)_{k=1, \cdots, 10}=\left[\begin{array}{r}
0.880436+0.580619 i \\
0.777887+1.413441 i \\
1.294131+3.904156 i \\
-2.267198-2.863950 i \\
3.496869-19.491173 i \\
0.880436-0.580619 i \\
0.777887-1.413441 i \\
1.294131-3.904156 i \\
-2.267198+2.863950 i \\
3.496869+19.491173 i
\end{array}\right]
$$

and

$$
\beta^{(5)}:=\left(\beta_{k}^{(5)}\right)_{k=1, \cdots, 10}=\left(\left(p_{k}, v_{5}\right)\right)_{k=1, \cdots, 10}=\left[\begin{array}{r}
0.375336-0.419733 i \\
-0.598245+0.653683 i \\
-0.663339-2.567674 i \\
1.476882-2.242916 i \\
14.979607+3.029025 i \\
0.375336+0.419733 i \\
-0.598245-0.653683 i \\
-0.663339+2.567674 i \\
1.476882+2.242916 i \\
14.979607-3.029025 i
\end{array}\right] .
$$

\subsection{Computational aspects}

In this subsection, we say something about the used computer equipment and the computation times.

(i) As to the computer equipment, the following hardware was available: an Intel Core2 Duo Processor at $3166 \mathrm{GHz}$, a $500 \mathrm{~GB}$ mass storage facility, and two $2048 \mathrm{MB}$ high-speed memories. As software package for the computations, we used MATLAB, Version 7.11.

(ii) The computation time $t$ of an operation was determined by the command sequence $t 1=$ clock; operation; $t=e t i m e(c l o c k, t 1)$. It is put out in seconds, rounded to four decimal places. For the computation of the eigenvalues of matrix $A$ in Subsection 8.3, we used the command $[\mathrm{XA}, \mathrm{DA}]=\mathrm{eig}(\mathrm{A})$; the pertinent computation time was less than $0.0001 \mathrm{~s}$.

\section{Numerical Example 2}

In this section, we proceed in a similar way as in Section 8. Here, we present an example of a real nonsymmetric matrix $A$ with real eigenvalues. 


\subsection{The matrix $A$ and its eigenvalues and eigenvectors}

We take the matrix $A$ from [1, Example 5.1, p.81]. So, let

$$
A=\left[\begin{array}{rrr}
33 & 16 & 72 \\
-24 & -10 & -57 \\
-8 & -4 & -17
\end{array}\right] \text {. }
$$

In [1], the eigenvalues are given as

$$
\begin{aligned}
& \lambda_{1}=3, \\
& \lambda_{2}=2, \\
& \lambda_{3}=1,
\end{aligned}
$$

where the numbering is such that $\lambda_{1} \geq \lambda_{2} \geq \lambda_{3}$. According to [1], the associated right eigenvectors are given as

$$
p_{1}=\left[\begin{array}{r}
-4 \\
3 \\
1
\end{array}\right], p_{2}=\left[\begin{array}{r}
-16 \\
13 \\
4
\end{array}\right], p_{3}=\left[\begin{array}{r}
-15 \\
12 \\
4
\end{array}\right] ;
$$

they are unnormed.

\subsection{Auxiliary computational results}

Using the Matlab routine eig.m, we obtain

$$
\begin{aligned}
& \lambda_{1}=3.000000000000039, \\
& \lambda_{2}=2, \\
& \lambda_{3}=0.999999999999961,
\end{aligned}
$$

as well as the pertinent computed biorthonormal right eigenvectors $p_{i}, i=1,2,3$ and $u_{j}^{*}, j=1,2,3$, i.e. with the property $\left(p_{i}, u_{j}^{*}\right)=\delta_{i j}, i, j=1,2,3$ as

$$
\begin{aligned}
& p_{1}=\left[\begin{array}{r}
0.784464540552736 \\
-0.588348405414552 \\
-0.196116135138184
\end{array}\right], u_{1}^{*}=\left[\begin{array}{r}
20.396078054371419 \\
20.396078054372179 \\
15.297058540776456
\end{array}\right], \\
& p_{2}=\left[\begin{array}{r}
0.761904761904761 \\
-0.619047619047621 \\
-0.190476190476190
\end{array}\right], u_{2}^{*}=\left[\begin{array}{r}
0.000000000002098 \\
-20.999999999997225 \\
62.999999999999801
\end{array}\right], \\
& p_{3}=\left[\begin{array}{r}
0.764470787156438 \\
-0.611576629725151 \\
-0.203858876575050
\end{array}\right], u_{3}^{*}=\left[\begin{array}{r}
-19.621416870349535 \\
0.000000000001163 \\
-78.485667481401293
\end{array}\right] .
\end{aligned}
$$

\subsection{Numerical check of Corollaries 15 and 17}

From Corollary 15, Formula (72) and Corollary 17, Formula (75), we conclude

$$
\min _{j=1,2,3} \lambda_{j}(A) \leq \frac{(A u, v)}{(u, v)} \leq \max _{j=1,2,3} \lambda_{j}(A),
$$

$(u, v) \gg 0, u \in N_{p, \mathbb{R}}=\left[p_{1}, p_{2}, p_{3}\right]_{\mathbb{R}}, v \in N_{u^{*}, \mathbb{R}}=\left[u_{1}^{*}, u_{2}^{*}, u_{3}^{*}\right]_{\mathbb{R}}$ by setting $k=1$, there. This can also be written as

$$
\frac{(A u, v)}{(u, v)} \in\left[\min _{j=1,2,3} \lambda_{j}(A), \max _{j=1,2,3} \lambda_{j}(A)\right],
$$


$(u, v) \gg 0, u \in N_{p, \mathbb{R}}, v \in N_{u^{*}, \mathbb{R}}$. We check this for a series of vectors. One has

$$
\left[\min _{j=1,2,3} \lambda_{j}(A), \max _{j=1,2,3} \lambda_{j}(A)\right]=[1 ; 3] .
$$

Let

$$
\begin{aligned}
& u_{1}=-5 p_{1}+3 p_{3}, \\
& v_{1}=-4 u_{1}^{*}+2 u_{3}^{*} .
\end{aligned}
$$

Then $u_{1} \in N_{p, \mathbb{R}}$ and $v_{1} \in N_{u^{*}, \mathbb{R}}$ as well as $\left(u_{1}, v_{1}\right) \gg 0$, and one obtains

$$
\begin{gathered}
u_{1}=\left[\begin{array}{r}
-1.628910341294369 \\
1.107012137897305 \\
0.369004045965768
\end{array}\right], \quad v_{1}=\left[\begin{array}{l}
-1.208271459581847 \\
-0.815843122174864 \\
-2.181595691259084
\end{array}\right] \times 10^{2}, \\
\left(A u_{1}, v_{1}\right)=66.000000000003695, \\
\left(u_{1}, v_{1}\right)=26.000000000001563,
\end{gathered}
$$

and thus

$$
\frac{\left(A u_{1}, v_{1}\right)}{\left(u_{1}, v_{1}\right)} \doteq 2.538461538461528 \in[1 ; 3] .
$$

Let

$$
\begin{array}{rrr}
u_{2}= & 3 p_{2}, \\
v_{2}= & -4 u_{4}^{*}+2 u_{2}^{*} .
\end{array}
$$

Then $u_{2} \in N_{p, \mathbb{R}}$ and $v_{2} \in N_{u^{*}, \mathbb{R}}$ as well as $\left(u_{2}, v_{2}\right) \gg 0$, and one obtains

$$
\begin{gathered}
u_{2}=\left[\begin{array}{r}
2.285714285714283 \\
-1.857142857142862 \\
-0.571428571428569
\end{array}\right], \quad v_{2}=\left[\begin{array}{r}
-0.815843122174815 \\
-1.235843122174832 \\
0.648117658368938
\end{array}\right] \times 10^{2}, \\
\left(A u_{2}, v_{2}\right)=12.000000000003737, \\
\left(u_{2}, v_{2}\right)=6.000000000001315,
\end{gathered}
$$

and thus

$$
\frac{\left(A u_{2}, v_{2}\right)}{\left(u_{2}, v_{2}\right)} \doteq 2.000000000000185 \in[1 ; 3] .
$$

Let

$$
\begin{aligned}
& u_{3}=-5 p_{1}+3 p_{2}-4 p_{3}, \\
& v_{3}=-4 u_{1}^{*}+2 u_{2}^{*}-2 u_{3}^{*} .
\end{aligned}
$$

Then $u_{3} \in N_{p, \mathbb{R}}$ and $v_{3} \in N_{u^{*}, \mathbb{R}}$ as well as $\left(u_{3}, v_{3}\right) \gg 0$, and one obtains

$$
\begin{gathered}
u_{3}=\left[\begin{array}{r}
-4.694491565675151 \\
3.530905688830501 \\
1.224587610562552
\end{array}\right], \quad v_{3}=\left[\begin{array}{r}
-0.423414784767824 \\
-1.235843122174855 \\
2.217831007996964
\end{array}\right] \times 10^{2}, \\
\left(A u_{3}, v_{3}\right)=80, \\
\left(u_{3}, v_{3}\right)=34.000000000000313,
\end{gathered}
$$

and thus

$$
\frac{\left(A u_{3}, v_{3}\right)}{\left(u_{3}, v_{3}\right)} \doteq 2.352941176470567 \in[1 ; 3] .
$$

Let

$$
\begin{array}{lll}
u_{4}= & -5 p_{1}+3 p_{2}, \\
v_{4}= & -2 u_{3}^{*} .
\end{array}
$$

Then $u_{4} \in N_{p, \mathbb{R}}$ and $v_{4} \in N_{u^{*}, \mathbb{R}}$, but $\left(u_{4}, v_{4}\right) \gg 0$, and one obtains

$$
u_{4}=\left[\begin{array}{r}
-1.636608417049400 \\
1.084599169929897 \\
0.409152104262350
\end{array}\right], \quad v_{4}=\left[\begin{array}{r}
0.392428337406991 \\
-0.000000000000023 \\
1.569713349628026
\end{array}\right] \times 10^{2} \text {, }
$$




$$
\begin{aligned}
\left(A u_{4}, v_{4}\right) & =-6.821210263296962 \times 10^{-13}, \\
\left(u_{4}, v_{4}\right) & =7.105427357601002 \times 10^{-14},
\end{aligned}
$$

and thus

$$
\frac{\left(A u, v_{4}\right)}{\left(u_{4}, v_{4}\right)}=-9.600000000000000 \notin[1 ; 3]
$$

which is not surprising since $\left(u_{4}, v_{4}\right) \approx 0$.

Let

$$
\begin{aligned}
& u_{5}=[2,3,4]^{T} \in \mathbb{R}^{3}, \\
& v_{5}=[4,3,2]^{T} \in \mathbb{R}^{3} .
\end{aligned}
$$

Here, one obtains

$$
\begin{aligned}
\left(A u_{5}, v_{5}\right) & =498 \\
\left(u_{5}, v_{5}\right) & =25
\end{aligned}
$$

and thus

$$
\frac{\left(A u_{5}, v_{5}\right)}{\left(u_{5}, v_{5}\right)}=19.920000000000002 \notin[1 ; 3]
$$

which is neither surprising since $\left(u_{5}, v_{5}\right) \ngtr 0$ due to

$$
\alpha^{(5)}:=\left(\alpha_{k}^{(5)}\right)_{k=1,2,3}=\left(\left(u_{5}, u_{k}^{*}\right)\right)_{k=1,2,3}=\left[\begin{array}{r}
1.631686244349652 \\
1.890000000000117 \\
-3.531855036663008
\end{array}\right] \times 10^{2}
$$

and

$$
\beta^{(5)}:=\left(\beta_{k}^{(5)}\right)_{k=1,2,3}=\left(\left(p_{k}, v_{5}\right)\right)_{k=1,2,3}=\left[\begin{array}{l}
0.980580675690923 \\
0.809523809523802 \\
0.815435506300197
\end{array}\right] .
$$

\section{Conclusion and outlook on to future work}

It has been shown that there exist generalized Rayleigh-quotient representations of the real and imaginary parts of the eigenvalues of diagonalizable matrices that parallel those for the eigenvalues of self-adjoint matrices. For the moduli, however, only a max-representation could be derived. The special case of diagonalizable matrices with real eigenvalues has also been considered. The key idea for the derivation of the new formulas is to use the subspaces $N_{p, \mathbb{R}}$ and $N_{u^{*}, \mathbb{R}}$ of $\mathbb{C}^{n}$ and the more general quotients $(A u, v) /(u, v),(u, v) \gg 0, u \in N_{p, \mathbb{R}}, v \in N_{u^{*}, \mathbb{R}}$. As application, a new formula for the spectral radius $\rho(A)$ is obtained. On a numerical example from the theory of linear dynamical systems (Example 1), we check that $\frac{\operatorname{Re}(A u, v)}{(u, v)} \in$ $\left[\min _{j=1, \cdots, n} \operatorname{Re} \lambda_{j}(A), \max _{j=1, \cdots, n} \operatorname{Re} \lambda_{j}(A)\right],(u, v) \gg 0, u \in N_{p, \mathbb{R}}, v \in N_{u^{*}, \mathbb{R}}$. On a further example (Example 2), this time for a matrix $A$ with real eigenvalues, we check numerically that $\frac{(A u, v)}{(u, v)} \in\left[\min _{j=1, \cdots, n} \lambda_{j}(A), \max _{j=1, \cdots, n} \lambda_{j}(A)\right],(u, v) \gg 0, u \in N_{p, \mathbb{R}}, v \in N_{u^{*}, \mathbb{R}}$. We mention that, in the case of self-adjoint matrices, both Section 2 and Section 5 deliver back the results of [7]. The paper is of interest on its own in the areas of Linear Algebra and Numerical Analysis. Beyond this, it could be of value to mathematicians and engineers.

The case of general matrices is more involved and will be dealt with in a subsequent paper. 


\section{References}

[1] R.T. Gregory, D.L. Karney, A Collection of Matrices for Testing Computational Algorithms, Wiley-Interscience, New York London Sydney Toronto, 1969.

[2] R.A. Horn, Ch.R. Johnson, Matrix Analysis, University Press, Cambridge, 22nd printing, 2009.

[3] R.A. Horn, Ch.R. Johnson, Topics in Matrix Analysis, University Press, Cambridge, 10th printing, 2008.

[4] T. Kato, Perturbation Theory for Linear Operators, Springer, New York, 1966.

[5] L. Kohaupt, Construction of a biorthogonal system of principal vectors of the matrices $A$ and $A^{*}$ with applications to the initial value problem $\dot{x}=A x, x\left(t_{0}\right)=x_{0}$, Journal of Computational Mathematics and Optimization 3(3)(2007)163-192.

[6] L. Kohaupt, Biorthogonalization of the principal vectors for the matrices $A$ and $A^{*}$ with application to the computation of the explicit representation of the solution $x(t)$ of $\dot{x}=A x, x\left(t_{0}\right)=x_{0}$, Applied Mathematical Sciences 2(20)(2008)961-974.

[7] L. Kohaupt, Generalized Rayleigh-quotient representation of the eigenvalues of self-adjoint matrices, Journal of Algebra and Applied Mathematics 14(1)(2016)1-26.

[8] L. Kohaupt, Rayleigh-quotient representation of the real parts, imaginary parts, and moduli of the eigenvalues of diagonalizable matrices, to be submitted for publication.

[9] F. Stummel, K. Hainer, Introduction to Numerical Analysis, Scottish Academic Press, Edinburgh, 1980.

[10] L.H. Wilkinson, The Algebraic Eigenvalue Problem, Clarendon Press, Oxford, 1965.

[11] R. Zurmühl, S. Falk, Matrizen und ihre Anwendungen, Teil 1: Grundlagen (Matrices and Their Applications, Part 1: Foundations), Springer-Verlag, Berlin Heidelberg New York Tokyo, 1984.

\section{Appendix}

In a Remark at the end of Section 4, we have indicated that, with a minor additional hypothesis, the generalized min-, minmax-, and maxmin-representaions for the moduli of eigenvalues can be proven. In this Appendix, we show this, but restrict ourselves to the min-representation. The minor additional hypothesis is $p_{j} \in M_{p}$ and $u_{j}^{*} \in M_{u^{*}}$. A further advantage of this additional hypothesis is that the proofs simplify.

We have

\section{Theorem 18:}

Let the conditions $(C 1)-(C 4)$ be fulfilled. Further, let the eigenvalues of $A$ be arranged according to (66).

Then, for every $j=1, \cdots, n$ and every subspace $M_{p} \subset N_{p, \mathbb{R}}$ and $M_{u^{*}} \subset N_{u^{*}, \mathbb{R}}$ with $\operatorname{dim} M_{p}=$ $\operatorname{dim} M_{u^{*}}=m=n+1-j$ where additionally $\mathbf{p}_{\mathbf{j}} \in \mathbf{M}_{\mathbf{p}}$ and $\mathbf{u}_{\mathbf{j}}^{*} \in \mathbf{M}_{\mathbf{u}^{*}}$, the following inequalities are valid:

$$
\left|\lambda_{j}(A)\right| \leq \max _{\substack{(u, v) \gg 0 \\ u \in M_{p}, v \in M_{u^{*}}}} \frac{|(A u, v)|}{(u, v)} \leq\left|\lambda_{1}(A)\right|,
$$

and the following representation formulas hold:

$$
\left|\lambda_{j}(A)\right|=\min _{\substack{\operatorname{dim} M_{p}=m, p_{j} \in M_{p} \\ \operatorname{dim} M_{u^{*}}=m, u_{j}^{*} \in M_{u^{*}}}} \max _{\substack{(u, v) \gg 0 \\ u \in M_{p}, v \in M_{u^{*}}}} \frac{|(A u, v)|}{(u, v)} .
$$

Proof: One has

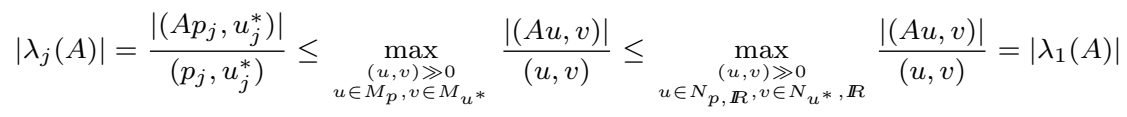

so that (91) is proven. Further, from (93)

$$
\left|\lambda_{j}(A)\right| \leq \min _{\substack{\operatorname{dim} M_{p}=m, p_{j} \in M_{p} \\ \operatorname{dim} M_{u^{*}}=m, u_{j}^{*} \in M_{u^{*}}}} \max _{\substack{(u, v) \gg 0 \\ u \in M_{p}, v \in M_{u^{*}}}} \frac{|(A u, v)|}{(u, v)} .
$$

On the other hand, from Theorem 13

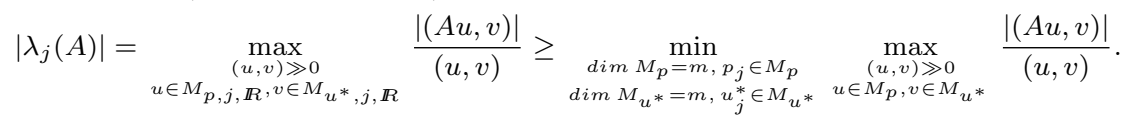

Relations (94) and (95) imply (92).

Remark: We mention that, with the above additional hypotheses, the proofs of Theorems $4-7$, Theorems 9 - 12, Theorem 13, and Corollaries 14 - 17 get also simpler. 